\title{
Article \\ Deterministic model of the eddy dynamics for a midlatitude ocean model
}

\author{
Takaya Uchida $^{1}\left(\mathbb{D}\right.$, Bruno Deremble ${ }^{1, t, *(\mathbb{D})}$ and Stephane Popinet ${ }^{2}(\mathbb{D}$ \\ 1 Université Grenoble Alpes, Centre National de la Recherche Scientifique, Institut des Géosciences de \\ l'Environnement \\ 2 Sorbonne Université, Centre National de la Recherche Scientifique, Institut Jean le Rond d'Alembert \\ * Correspondence: bruno.deremble@cnrs.fr \\ † Current address: Maison Climat-Planete, 460 Rue de la Piscine, 38400 Saint-Martin d'Hères, France
}

\begin{abstract}
Mesoscale eddies, the weather system of the oceans, although being on the scales of $O(20-100 \mathrm{~km})$, have a disproportionate role in shaping the mean jets such as the separated Gulf Stream in the North Atlantic Ocean, which is on the scale of $O(1000 \mathrm{~km})$ in the along-jet direction. With the increase in computational power, we are now able to partially resolve the eddies in basinscale and global ocean simulations, a model resolution often referred to as mesoscale permitting. It is well known, however, that due to grid-scale numerical viscosity, mesoscale permitting simulations have less energetic eddies and consequently weaker eddy feedback onto the mean flow. In this study, we run a quasi-geostrophic model at mesoscale resolving resolution in a double gyre configuration and formulate a deterministic parametrization for the eddy rectification term of potential vorticity (PV), namely, the eddy PV flux divergence. We have moderate success in reproducing the spatial patterns and magnitude of eddy kinetic and potential energy diagnosed from the model. One novel point about our approach is that we account for non-local eddy feedbacks onto the mean flow by solving the eddy PV equation prognostically in addition to the mean flow. In return, we are able to parametrize the variability in total (mean+eddy) PV at each time step instead of solely the mean PV. A closure for the total PV is beneficial as we are able to account for both the mean state and extreme events.
\end{abstract}

Keywords: Mesoscale eddy closure; Quasi geostrophy; Mid-latitude double gyre

\section{Introduction}

In the field of fluid dynamics and turbulence, formulating a closure for the governing equations has been a long standing problem [1,2]. This is because there is often not enough resolution whether it be an observational or modelling study to resolve the variable of interest down to the molecular scale where kinetic energy is dissipated to internal energy due to molecular viscosity. Particularly in the field of geophysical fluid dynamics (GFD) where the scales of interest span up to $O(1000 \mathrm{~km})$, resolving the molecular scale let alone three-dimensional turbulence $[O(10 \mathrm{~m})$; 3$]$ is practically unachievable and will remain so for the foreseeable future. Due to the lack of resolution, the governing equations for the "resolved" field have an additional forcing term from the "unresolved" field. In other words, the governing equations are not closed. A large effort in GFD has been, therefore, to formulate a closure for the unresolved field, i.e. represent the unresolved field prognostically with the resolved momentum and/or tracer field [e.g. 4-7].

The fact that the unresolved (small-scale) field not only drains energy from the resolved (large-scale) field but also partially feeds back onto the resolved field by fluxing momentum and buoyancy back into the latter has been known for some time [8-10]. More recently, this inverse cascade of momentum from small to large scale has gained serious attention in the ocean modelling community. This has partially been due to 
us not having the computational power until the last decade to partially resolve the mesoscale $O(20-100 \mathrm{~km})$ eddies on a global scale. The ocean currents are most energetic in the mesoscale range [11-14]. Modelling studies with varying spatial resolution have shown that only partially resolving the mesoscale results in weaker mesoscale eddies, and consequently weaker feedback onto large-scale flows such as the Gulf Stream $[15,16]$. Considering the impact of the mean jets on global tracer transport and air-sea interaction [17-20], improving the representation of the eddy feedback onto the mean flow has climate implications. There has been a growing effort, therefore, to represent the inverse cascade of kinetic energy otherwise lost to grid-scale numerical viscosity at mesoscale permitting resolution, a process often referred to as energy backscattering parametrizations [e.g. 21-27, and references therein]. Our study here is in the same realm of parametrization studies in which we aim to improve the large-scale state by parametrizing the net mesoscale feedback onto the former.

Specifically, the goal of our study is to formulate a deterministic closure and hence a model for the eddy dynamics. Such approach is not new; for example, Jansen et al. [23], Juricke et al. [25] and Perezhogin [26] implement a prognostic equation for the sub-grid (unresolved) eddy energy and achieve the backscattering via a negative viscosity. One notable difference in our method is that while many previous studies have formulated their parametrizations based on a local closure (i.e. relating the eddy momentum flux locally at each grid point to the resolved momentum), we construct our closure by incorporating basin-scale information. This is motivated by the fact that Venaille et al. [28] and Grooms et al. [29] have shown that the eddy feedback on the large-scale flow is strongly non-local. We also focus on the eddy potential vorticity (PV) equation rather than eddy energy within the quasi-geostrophic (QG) framework. The QG framework has been shown to be fruitful in examining the eddy-mean flow interaction and formulating eddy closures [e.g. 22,30-32]. Here, we propose a spatial filtering approach to achieve a PV-based deterministic closure.

The paper is organized as follows: We describe our QG model configuration in section 2 and in particular the eddy PV model in section 2.2. In depth analysis of the eddy model is given in section 3 and details on the spatial filtering are in section 4 . We give our conclusions in section 5 .

\section{Model and methods}

\subsection{Description of the model}

We adopt the QG framework in order to describe the well known double gyre circulation in an idealized midlatitude ocean basin. This model is known to capture both the large-scale and small-scale variability of the ocean with a relatively coarse vertical resolution [cf. 33]. The QG formalism is meant to describe dynamical regimes for a prescribed background stratification $N^{2}$ and Coriolis parameter $f$. Two ingredients are necessary to reproduce the double gyre pattern: the planetary vorticity must vary with latitude and we need to use a cyclonic forcing in the northern part of the domain and an anticyclonic forcing in the southern part of the domain. In order to satisfy the first condition, we work with the $\beta$-plane approximation such that the Coriolis parameter $f$ varies linearly with latitude. This sets the planetary scale $L_{\beta}=f_{0} / \beta$ which is large compared to the deformation scale $N H / f_{0}$, (with $H$ the depth of the ocean and $f_{0}$ the average value of the Coriolis parameter in the domain). In this formalism, the main dynamical variable is the quasi-geostrophic potential vorticity defined as

$$
q=\nabla^{2} \psi+\Gamma \psi \stackrel{\text { def }}{=} \mathcal{L} \psi,
$$

with $\psi$ the stream function, $\nabla^{2}$ the horizontal Laplace operator and

$$
\Gamma \stackrel{\text { def }}{=} \frac{\partial}{\partial z} \frac{f_{0}^{2}}{N^{2}} \frac{\partial}{\partial z}
$$


the vertical stretching operator. The horizontal velocity is defined as

$$
u=-\frac{\partial \psi}{\partial y} \quad \text { and } \quad v=\frac{\partial \psi}{\partial x},
$$

and the buoyancy is defined as

$$
b=f_{0} \frac{\partial \psi}{\partial z} .
$$

The equation of evolution of the potential vorticity is

$$
\frac{\partial q}{\partial t}+J(\psi, q)+\beta v=A_{4} \nabla^{4} q+r_{b} \nabla^{2} \psi+F,
$$

with

$$
J(a, b)=\frac{\partial a}{\partial x} \frac{\partial b}{\partial y}-\frac{\partial a}{\partial y} \frac{\partial b}{\partial x},
$$

the jacobian operator, which corresponds to the non linear advective term, $A_{4}$ the biharmonic viscosity, $r_{b}$ the bottom friction coefficient which parameterizes a bottom Ekman layer (and is thus non zero in the lower layer only), and $F$ the forcing resulting from an Ekman pumping in a thin Ekman layer at the surface and is thus non zero in the upper layer only. We build the numerical version of this model in the basilisk framework $[34,35]$.

We solve Eqs. (5) and (1) in a horizontal square domain with side $L=5000 \mathrm{~km}$ and of vertical extension $H=5000 \mathrm{~m}$. We discretize these equations with $512 \times 512$ horizontal points (which correspond to a horizontal resolution of slightly less than $10 \mathrm{~km}$ ) and 4 vertical layers of thickness $h_{1}=238 \mathrm{~m}, h_{2}=476 \mathrm{~m}, h_{3}=953 \mathrm{~m}$ and $h_{4}=3333 \mathrm{~m}$ (from top to bottom). We adjust the background stratification $N^{2}$ to mimic the stratification in middle of the subtropical gyre in the North Atlantic such that at each layer interface, we have $N_{1.5}^{2}=1.7 \times 10^{-5} \mathrm{~s}^{-2}, N_{2.5}^{2}=1.1 \times 10^{-5} \mathrm{~s}^{-2}$, $N_{3.5}^{2}=3.2 \times 10^{-7} \mathrm{~s}^{-2}$, from top to bottom. The average value of the Coriolis parameter is $f_{0}=9.3 \times 10^{-5} \mathrm{~s}^{-1}$ and $\beta=1.7 \times 10^{-11} \mathrm{~m}^{-1} \mathrm{~s}^{-1}$. For these parameters, the three deformation radii are $R_{d 1}=25 \mathrm{~km}, R_{d 2}=10 \mathrm{~km}$ and $R_{d 3}=7 \mathrm{~km}$. Note that these deformation radii correspond to the inverse squared eigenvalue of the vertical stretching operator. At this resolution we choose $A_{4}=6.25 \times 10^{9} \mathrm{~m}^{4} \mathrm{~s}^{-1}$, and $\delta_{e}=7.5 \mathrm{~m}$ (such that the spindown time scale is $1 / r_{b}=166$ days.

We solve the elliptic equation (Eq. 1) with homogeneous dirichlet boundary conditions on the sides ( $\psi=0$ which correspond to no flux boundary condition) and homogeneous Neumann boundary conditions at the top and bottom boundary (which correspond to the traditional QG assumption: $b=0$ at the upper and lower boundary).

The forcing is

$$
F=\frac{\nabla \times \tau}{\rho_{0} h_{1}}, \quad \text { with } \quad \tau=\tau_{0} \sin ^{3}\left(\frac{\pi y}{L}\right) .
$$

We use a cubic sine function in the definition of the wind in order to reproduce a narrow midlatitude atmospheric jet. For such a narrow jet, the boundary between the positive and negative area of the wind stress curl pattern is sharper than if we use the traditional cosine shape for the wind pattern. We choose $\tau_{0}=0.25 \mathrm{~N} \mathrm{~m}^{-2}$ which is an acceptable value for the difference between the maximum and minimum value of the wind in the North Atlantic ${ }^{1}$ [36]. We have also kept the wind stress axisymmetric as our interest is on eddy time scales and not low frequency variability.

To integrate the model in time, we first perform a spin up phase of 80 years at low resolution $(80 \mathrm{~km})$ followed by another 80 years at the prescribed resolution $(10 \mathrm{~km})$. After this spin up, the model is in a statistically steady state.

1 Note that this wind pattern takes only positive values: we could have added a term $-\tau_{0} / 2$ in the definition of the wind in Eq. (7), however this is only cosmetic because this additional term does not impact the wind stress curl, which is what ultimately matters in QG dynamics. 


\section{2.2. Mean flow and eddy models}

We decompose each dynamical variable as the sum of its time mean (denoted with an overbar) and a perturbation (denoted with a prime) as shown here for the stream function

$$
\psi=\bar{\psi}+\psi^{\prime}
$$

If we use this decomposition in the equation of evolution of PV, we get

$$
\frac{\partial}{\partial t}\left(\bar{q}+q^{\prime}\right)+J\left(\bar{\psi}+\psi^{\prime}, \bar{q}+q^{\prime}\right)+\beta\left(\bar{v}+v^{\prime}\right)=A_{4} \nabla^{4}\left(\bar{q}+q^{\prime}\right)+r_{b} \nabla^{2}\left(\bar{\psi}+\psi^{\prime}\right)+F,
$$

and if we take the time average of this equation, we get

$$
\frac{\partial \bar{q}}{\partial t}+J(\bar{\psi}, \bar{q})+\overline{J\left(\psi^{\prime}, q^{\prime}\right)}+\beta \bar{v}=A_{4} \nabla^{4} \bar{q}+r_{b} \nabla^{2} \bar{\psi}+F
$$

with $\bar{F}=F$ because we have a stationary forcing. The term $\overline{J\left(\psi^{\prime}, q^{\prime}\right)}$ is known as the eddy rectification of the large-scale flow. It is this term that many studies seek to parameterize [e.g. $22,30,32,37,38]$. In the present study, we are going to explicitly model the eddy dynamics with an independent model in order to compute this term. We obtain the eddy equation by taking the difference between Eq. (9) and Eq. (10)

$$
\frac{\partial q^{\prime}}{\partial t}+J\left(\psi^{\prime}, q^{\prime}\right)+J\left(\bar{\psi}, q^{\prime}\right)+J\left(\psi^{\prime}, \bar{q}\right)+\beta v^{\prime}=A_{4} \nabla^{4} q^{\prime}+r_{b} \nabla^{2} \psi^{\prime}+\overline{J\left(\psi^{\prime}, q^{\prime}\right)}
$$

Note that there is no explicit wind forcing in this equation: the forcing is present implicitly in the background time-mean flow. Note also that the term $\overline{J\left(\psi^{\prime}, q^{\prime}\right)}$ also appears in the eddy equation. This is somewhat cumbersome because to simulate the eddy model requires an a priori knowledge of the eddy rectification terms as a forcing which renders the eddy model meaningless. Although it may seem overkill here, it will become painful around section 3.2 to keep track of the eddy rectification terms, which appear upon both sides of Eq. (11) upon taking the time mean, so we will denote the rectification term on the right-hand side as $\mathcal{R}^{\prime}$ to distinguish its role as a forcing term. In the remainder of the study, the expression $\overline{J\left(\psi^{\prime}, q^{\prime}\right)}$ will be reserved for the rectification term diagnosed from the full model (Eq. 5) or the left-hand side of Eq. (11). We are going to propose a strategy to run this deterministic model of the eddy dynamics (Eq. 11): to perform a scale decomposition of the PV equation and assume that the eddy field corresponds to the small-scale flow (section 4).

\subsection{Energy diagnostics}

We analyze our simulation with energy diagnostics. In quasi geostrophy, the total energy is the sum of potential energy

$$
P E=\frac{1}{2} \frac{b^{2}}{N^{2}}
$$

and kinetic energy

$$
K E=\frac{1}{2}\left(u^{2}+v^{2}\right) .
$$

and since potential and kinetic energies are quadratic quantities, we write their time average as

$$
\begin{gathered}
\overline{P E}=\frac{1}{2} \frac{\bar{b}^{2}}{N^{2}}+\frac{1}{2} \frac{\overline{b^{\prime 2}}}{N^{2}} \stackrel{\text { def }}{=} \overline{\overline{P E}}+\overline{P E^{\prime}} \\
\overline{K E}=\frac{1}{2}\left(\bar{u}^{2}+\bar{v}^{2}\right)+\frac{1}{2}\left(\overline{u^{\prime 2}}+\overline{v^{\prime 2}}\right) \stackrel{\text { def }}{=} \overline{\overline{K E}}+\overline{K E^{\prime}},
\end{gathered}
$$


with $\overline{\overline{P E}}$ and $\overline{\overline{K E}}$ the potential and kinetic energy of the time mean flow and $\overline{P E^{\prime}}$ and $\overline{K E^{\prime}}$ the mean potential and kinetic energy of the eddy flow.

\subsection{Notation}

In the remainder of this paper, we adopt the following convention: we write with a prime (e.g. $\left.\psi^{\prime}\right)$, the diagnosed eddy field from the full model (Eq. 5), and with a dagger (e.g. $\left.\psi^{\dagger}\right)$ the prognostic eddy dynamics that result from the explicit time integration of the eddy model (Eq. 11) with the mean flow $(\bar{\psi}, \bar{q})$ as the input. Our aim is to build an eddy model for which $\overline{P E^{\dagger}}$ and $\overline{K E^{\dagger}}$ the potential and kinetic energies in the eddy flow mimic $\overline{P E^{\prime}}$ and $\overline{K E^{\prime}}$, the diagnosed eddy energies.

\subsection{Mean flow and eddy dynamics in the full model}

We first analyze the 80 years integration of the full model (Eq. 5). The stream function exhibits a standard double gyre pattern with an strong eddying jet that separate the cyclonic and anticylconic gyres. Such pattern has already been observed and described in numerous studies; we wish however to revisit it from an energetic perspective. We plot in Fig. 1a a snapshot of the eddy kinetic energy in the upper layer. We find at least two distinct dynamical regimes: (i) the eddying jet with $K E^{\prime}$ on the order of $0.5 \mathrm{~m}^{2} \mathrm{~s}^{-2}$ (corresponding to a velocity of $\left|u^{\prime}\right| \sim 1 \mathrm{~m} \mathrm{~s}^{-1}$ ). The intensity of the jet decreases downstream (eastward). (ii) a region with moderate eddies in the middle of each gyre; the magnitude of these eddies increases from East to West but their overall intensity is order $K E^{\prime} \sim 0.04 \mathrm{~m}^{2} \mathrm{~s}^{-2}\left(\left|u^{\prime}\right| \sim 0.2 \mathrm{~m} \mathrm{~s}^{-1}\right)$. There are other dynamical regions such as quiescent zone with no eddies at all at the same latitude as the jet but near the eastern boundary, and the regions near the northern and southern boundaries.

We plot with the same colorbar the eddy potential energy for the same snapshot (Fig. 1b). We observe that the magnitude of $P E^{\prime}$ is similar to the magnitude of $K E^{\prime}$ consistent with the QG scaling. We plot in Figs. 1c and $1 \mathrm{~d}$ the mean eddy kinetic energy and mean eddy potential energy. Both the eddy potential energy and eddy kinetic energy are maximum in the jet area and both fields exhibit very similar pattern. The maximum value of eddy energy in the jet area reflects the meandering jet. These meanders are strongest near the western boundary and decrease in amplitude as we go east.

The energy stored in the mean flow exhibits a radically different pattern than the eddy energy (Figs. 1e and 1f). The QG model exhibit the standard result that most of the large-scale energy is stored in the form of potential energy and only a small fraction of large-scale energy is stored in the form of kinetic energy. Note that the colorbar in Fig. If is extended by a factor 20 compared to the other plots because there is approximately 20 times more potential energy than kinetic energy in the large-scale flow. This result corresponds to the traditional view of the ocean circulation, although in our case both the large-scale and small-scale dynamics are handled by QG dynamics. In Fig. 1f, we see the bowl shape of the anticylconic gyre in the southern part of the domain (and respectively the dome shape of the cyclonic gyre in the northern part of the domain). Potential energy is maximum in the middle of the gyre where the buoyancy anomaly is maximum. The mean jet is much less energetic as shown in the kinetic energy panel (Fig. 1e). To summarize, we have $\overline{\overline{P E}} \gg \overline{P E^{\prime}} \sim \overline{K E^{\prime}}>\overline{\overline{K E}}$.

\subsection{Vorticity balance of the mean flow}

For sufficiently long integration, the first term in the mean flow (Eq. 10) will eventually vanish. There is thus a balance between the remaining terms of the mean PV equation. We only focus here on the rectification term that we plot in Fig. 2. We plot in Fig. 2a the raw estimate of this term $\left(\overline{J\left(\psi^{\prime}, q^{\prime}\right)}\right)$ computed with 500 independent snapshots that are 60 days apart, and in Fig. $2 \mathrm{~b}$ the smoothed version where we average 16 neighboring grid points and linearly interpolate back on the fine grid for visualization purposes. From the latter plot, a large-scale component of this field that emerges in the return flow area. The region of the separated jet exhibits a stronger signal whereas 
(a) $K E^{\prime}$

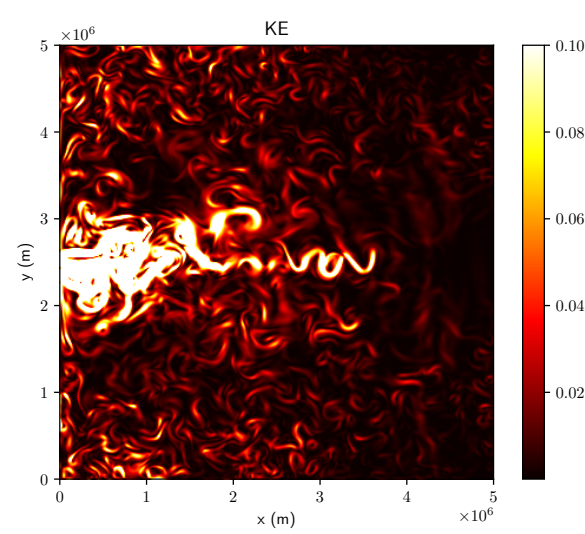

(c) $\overline{K E^{\prime}}$

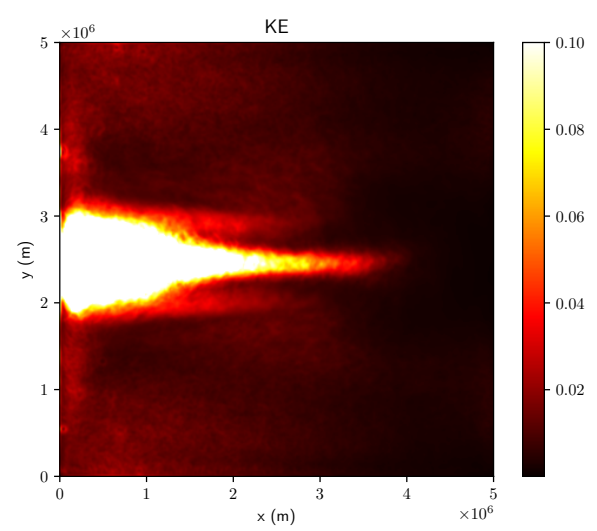

(e) $\overline{\overline{K E}}$

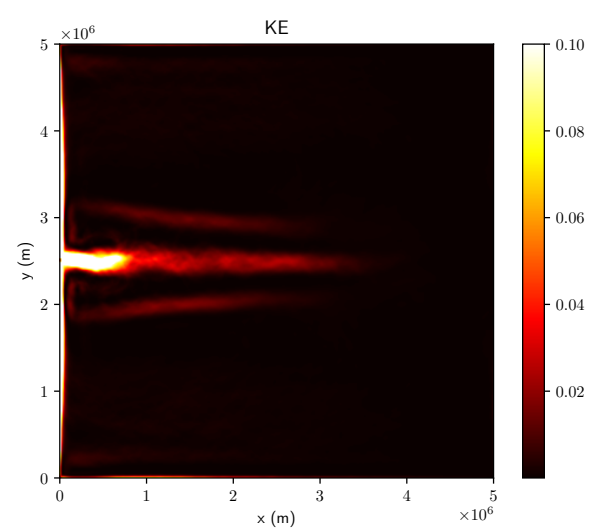

(b) $P E^{\prime}$

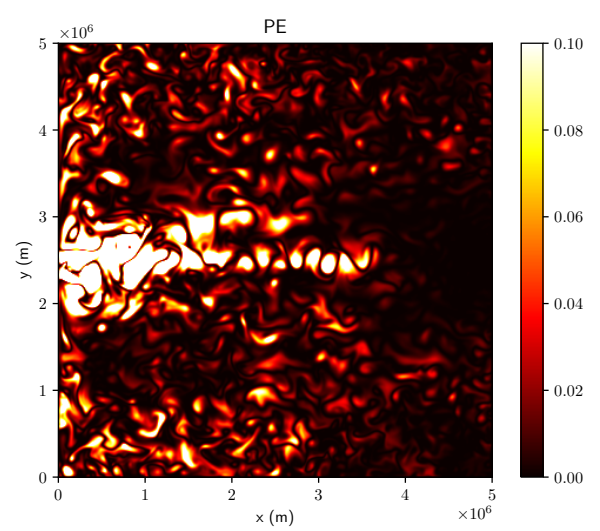

(d) $\overline{P E^{\prime}}$

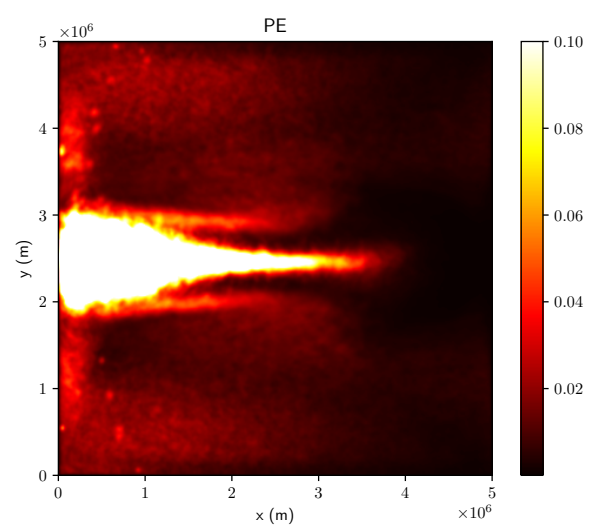

(f) $\overline{\overline{P E}}$

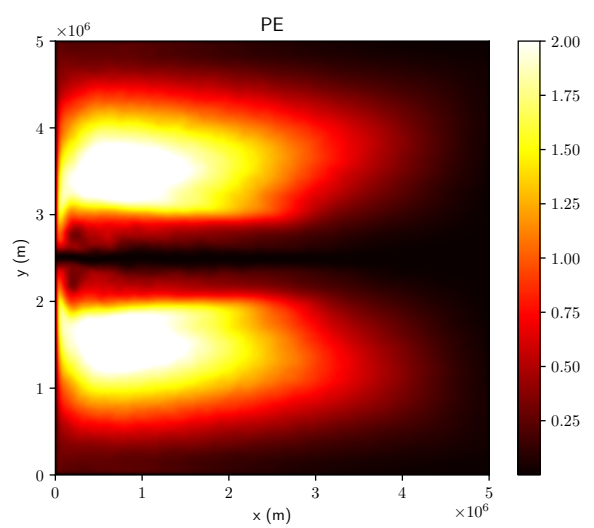

Figure 1. Snapshots and time-mean of potential energy and kinetic energy diagnosed from the full model. Units: $\mathrm{m}^{2} \mathrm{~s}^{-2}$ 
the region near the boundaries also exhibit intense magnitude signal. The pattern in Fig. 2a clearly has not converged because when we sum all the terms in Eq. (10), viz. $J(\bar{\psi}, \bar{q})+\overline{J\left(\psi^{\prime}, q^{\prime}\right)}+\beta \bar{v}-A_{4} \nabla^{4} \bar{q}-r_{b} \nabla^{2} \bar{\psi}-F$, we get a field with similar bittiness to Fig. 2a, whereas we should actually get zero everywhere if the model were run long enough $\left(\frac{\partial \bar{q}}{\partial t} \sim 0\right.$; not shown). With the purpose of formulating a deterministic model for the eddy rectification term, some spatial smoothing is appropriate in order to filter out stochastic variability. If we admit that the smoothed $\overline{J\left(\psi^{\prime}, q^{\prime}\right)}$ is the deterministic part and that $\overline{J\left(\psi^{\prime}, q^{\prime}\right)}$ should converge towards its smoothed version, we can estimate the number of samples we need for convergence with a maximum of $10 \%$ error. Indeed the standard error of the mean is given by $\sigma / \sqrt{n}$ where $\sigma$ is the standard deviation of the time series at a given point and $n$ the number of samples. If we want the errorbar to be $10 \%$ of the value of the mean $m$, the $95 \%$ confidence interval on the mean for that tolerance is given by $0.1 \mathrm{~m}=2 \sigma / \sqrt{n}$ such that $n=400 \sigma^{2} / \mathrm{m}^{2}$. We get an estimate of $n=10^{5}$ samples to get this $10 \%$ precision for the mean. This corresponds to $10^{4}$ years of simulation which is clearly out of reach in the current setup. We have tested this using the 2740 years of output from Kondrashov and Berloff [39] and found the convergence to be very slow (personal communication with Pavel Berloff). The fact that such a long integration is required for accurate statistics is problematic from an eddy closure perspective, namely the eddy statistics of today would depend on the dynamical state of the system thousands of years into the future.

(a) $\operatorname{Raw} \overline{J\left(\psi^{\prime}, q^{\prime}\right)}$

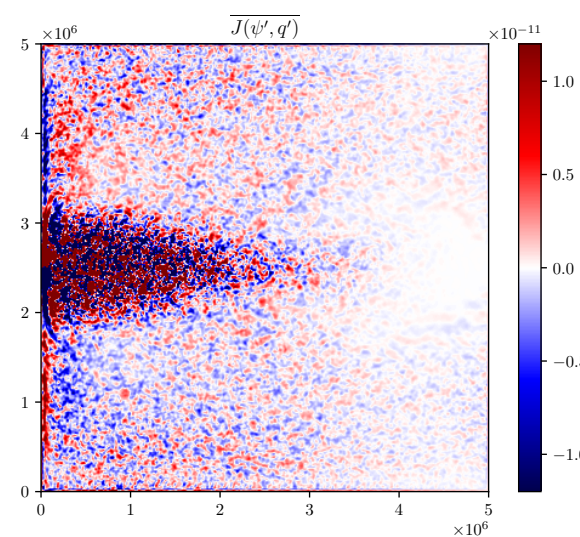

(b) Smoothed $\overline{J\left(\psi^{\prime}, q^{\prime}\right)}$

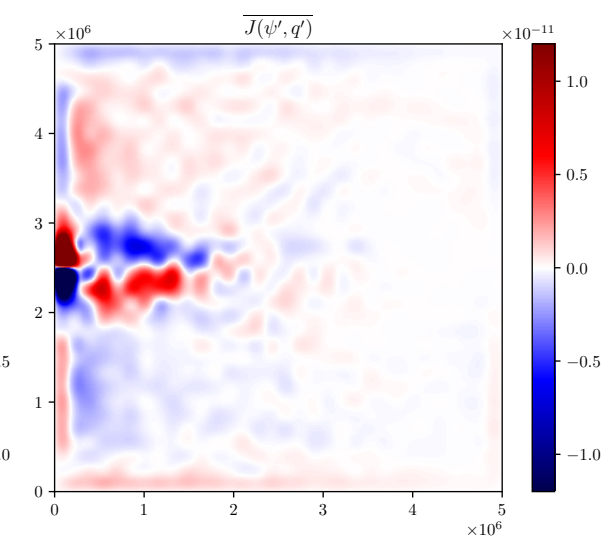

Figure 2. $\overline{J\left(\psi^{\prime}, q^{\prime}\right)}$ and $\overline{J\left(\psi^{\prime}, q^{\prime}\right)}$ smoothed by averaging 16 neighboring grid points and linearly interpolated back on the fine grid.

\section{Analysis of the small-scale model}

We now use the mean field of the run that we just described to force the perturbation equation (Eq. 11). As a preliminary sanity check, we perform a linear stability analysis of that background flow and then do the non-linear integration of the perturbation model.

\subsection{Linear stability analysis}

We first perform a linear stability analysis of the mean state that we described in the previous section. Methods to perform such analysis have been reported elsewhere [e.g. $8,13,40,41]$ and we only recall the main steps here. From the perturbation equation (Eq. 11), we drop the non-linear advective term as well as the rectification term and replace $\psi^{\prime}$ by one Fourier component

$$
\psi^{\prime}=\widehat{\psi}^{\prime}(z) \exp [i(k x+l y-\omega t)]+c c,
$$


where $c c$ stands for complex conjugate. For each Fourier component, we get an equation with four unknown: $\widehat{\psi}^{\prime}(z), k, l$, and $\omega$, respectively the vertical structure of the Fourier mode, the zonal, meridional, and temporal wave number. We span the $(k, l)$ space in order to find $\widehat{\psi^{\prime}}(z)$ and $\omega$, which are the eigenvector and the eigenvalue of the equation. If the imaginary part of $\omega$ is negative, the corresponding mode is exponentially decaying and the solution is stable but if the imaginary part of $\omega$ is positive, the solution is unstable. In the $(k, l)$ space, the most unstable mode corresponds to the solution for which $\operatorname{Im}(\omega)$ is maximum. We call

$$
\mathscr{T}=\frac{1}{\max _{(k, l)}(\operatorname{Im}(\omega))}
$$

the inverse growth rate of the most unstable mode, $k_{m}$ and $l_{m}$, the zonal and meridional wavelength of that most unstable mode, and

$$
\lambda=\frac{2 \pi}{\sqrt{k_{m}^{2}+l_{m}^{2}}},
$$

the length scale of that mode. We plot $\mathscr{T}$ and $\lambda$ in Fig. 3. One first important information from these plots is that the large-scale solution is unstable almost everywhere in the domain (except in the small white area at $y=2500 \mathrm{~km}$ near the eastern boundary). This was not obvious a priori because we computed the most unstable mode with the same viscosity as the full model and viscosity is known to damp instabilities. We divide the time scale pattern into three distinct dynamical regimes: the western boundary and the intergyre jet which have the fastest growing mode (order 20 days), the return flow near the northern and southern boundary for which the instability time scale is order 60 days, and the rest of the domain for which the instability time scale is greater than 115 days (the colorbar saturates beyond this value). We do not consider the instability with long time scale because such long time scale is much bigger than the eddy time scale and become irrelevant for the eddy dynamics (local instability analysis is probably not relevant in areas with such long time scales). The instability length scale is noisier but overall in the area where $\mathscr{T}<115$ days, the length scale of the instability is 10 times the deformation radius [consistent with the canonical 2-layer baroclinic instability; 42].

(a) $\mathscr{T}$ (days)

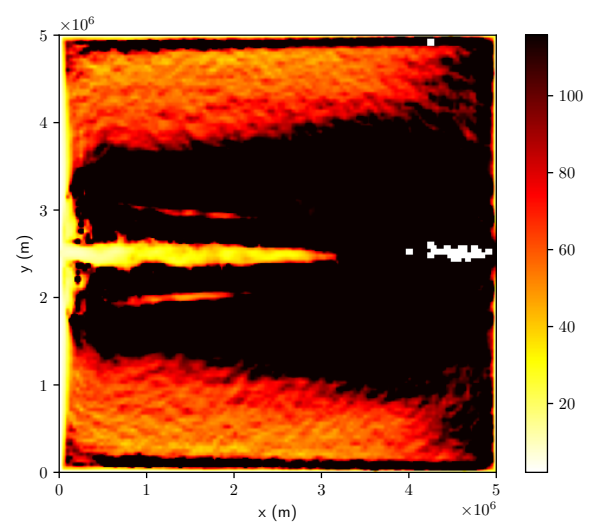

(b) $\lambda(\mathrm{km})$

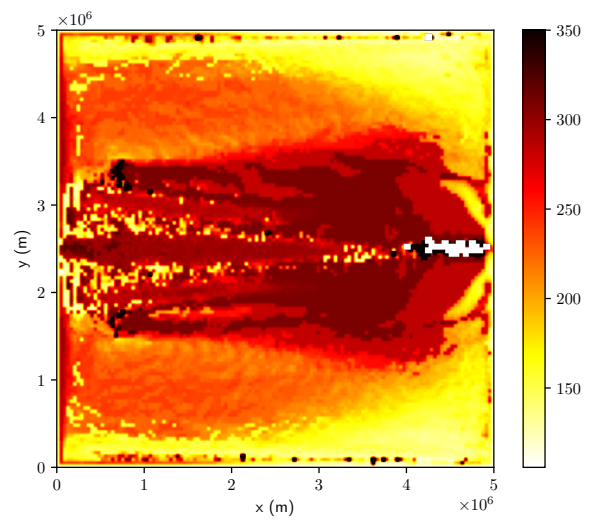

Figure 3. Time scale and length scale of the most unstable mode (compute every 4 grid point of the computational domain)

When we compare these plot with Fig. 1c, there does not seem to be an obvious link between the local instability parameter and the observed eddy kinetic energy. The path of the jet has a wider signature in the $\overline{K E^{\prime}}$ map. The demarcation between the return flow and the rest of the gyre that we observe in Fig. 3a also does not show up in the 
kinetic energy map. This confirms the conclusion of Grooms et al. [29] who showed that the eddies observed at one given location are mostly not locally generated but emanate from areas afar [see also 28]. We will return to these instability maps in section 4 on spatial filtering.

\subsection{Non linear run of the eddy model with no forcing}

Perhaps more interesting is the analysis of the non-linear simulation of the eddy model (Eq. 11) without the eddy rectification term on the right-hand side (viz. $\mathcal{R}^{\dagger}=0$ ). We recall that this equation has mostly been used to simulate local turbulence in doublyperiodic patches of the ocean with uniform shear [e.g. 28,29], whereas we now apply and solve this equation prognostically in the entire domain with a large-scale flow that varies in space. In other words, we will be examining the dagger variables (e.g. $\left.\psi^{\dagger}\right)$ where the primes in Eq. (11) are replaced by daggers.

For white noise initial conditions, we can decompose the run in several stages: we first observe a linear growth of the most unstable modes mainly in the jet and near the northern and southern boundary. The duration of this phase is on the same order of magnitude as the inverse linear growth rate, in agreement with the analysis done in the previous paragraph. We then enter another transient phase during which a large-scale pattern emerges in the PV field, and after this transient phase, we reach a statistical steady state. To illustrate this last regime, we plot in Fig. 4 the mean potential and kinetic energy as well as snapshot of these two fields. There are several important things to notice: first we note that $\overline{P E^{\dagger}}$ (Fig. $4 \mathrm{~d}$ ) is very different from $\overline{P E^{\prime}}$ (Fig. 1d): $\overline{P E^{\dagger}}$ is maximum along the western boundary and does not really reflect the eddies that were present in the jet in the reference run. In fact when we look at a snapshot of potential energy (Fig. 4b), we see that this potential energy field is the sum of a large-scale and small-scale flow.

Everywhere in the domain, the mean kinetic energy in this perturbation run (Fig. 4c) is weaker than the mean eddy kinetic energy diagnosed from the reference run (Fig. 1c), viz. $\overline{K E^{\dagger}}<\overline{K E^{\prime}}$. The lower energy levels in eddy kinetic and potential energy is also apparent in the isotropic wavenumber spectra (Fig. 5; compare the black solid and dotted lines). We compute the eddy kinetic and potential energy spectra $\left(\frac{\overline{\left.\hat{u}\right|^{2}}}{2}\right.$ and $\frac{\overline{|\hat{b}|^{2}}}{2 N^{2}}$ respectively where $(\hat{\cdot})$ is the Fourier transformed amplitude) over the whole domain of the first layer using the xrft Python package [43] and taper the fields with the Hann window as is commonly done when computing the spectra $[13,14,44]$. The periodogram is computed every 23 days over the last 580 days of output and then averaged. In the perturbation run, we still see a local kinetic energy $\left(K E^{+}\right)$maximum in the middle of the domain where the mean jet is and we also observe deformation radius size eddies in the rest of the gyre (Fig. 4a). Such difference between $\overline{P E^{\dagger}}$ and $\overline{K E^{\dagger}}$ where we see larger scale patterns in the former indicates that in this perturbation run, energy is stored in the large-scale buoyancy field rather than in small-scale eddies. We interpret these energy maps in the light of the inverse cascade in quasi geostrophy that fluxes energy toward larger scales $[8,45]$. Because of this inverse cascade, we see the apparition of a large-scale pattern superimposed on top of the prescribed large-scale circulation (i.e. $\bar{\psi}$ and $\bar{q}$ in Eq. 11). The sum of these two large-scale solutions as we see in Fig. 4d corresponds to a less baroclinically unstable state and hence weaker eddies (see Fig. 4a).

We also plot in Fig. 6a the eddy stream function for the same snapshot as the one plotted in Fig. 1, and in Fig. 6b the eddy stream function of the eddy model for the same snapshot as in Fig. 4. This plot confirms the differences already highlighted of a weaker baroclinicity in the perturbation run and also shows that large-scale Rossby waves present in the eddy field diagnosed from the full model ( $\psi^{\prime}$; Fig. 6a) are not present in the eddy model $\left(\psi^{\dagger} ;\right.$ Fig. $\left.6 \mathrm{~b}\right)$. This implies that the Rossby waves are excited by the winds ( $F$ in Eq. 9 ), which project themselves onto the temporally varying fields of $\psi^{\prime}$, whereas the eddy model $\left(\psi^{\dagger}\right)$ has no input to excite such waves. 
(a) $K E^{\dagger}$

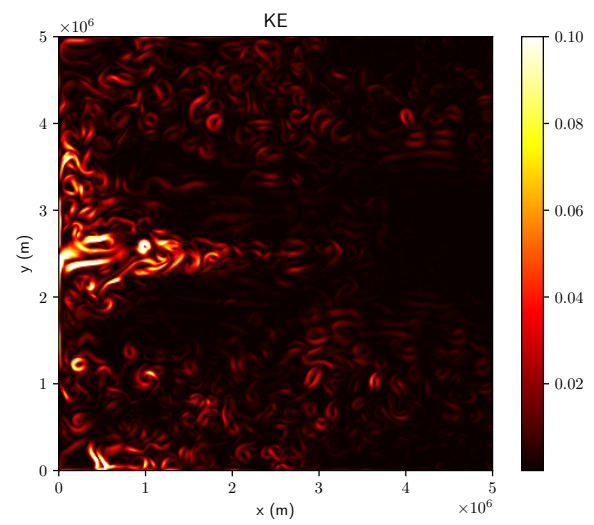

(c) $\overline{K E^{\dagger}}$

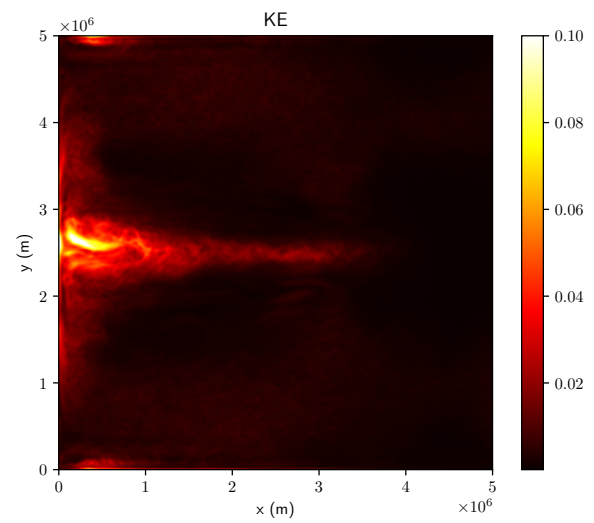

(b) $P E^{\dagger}$

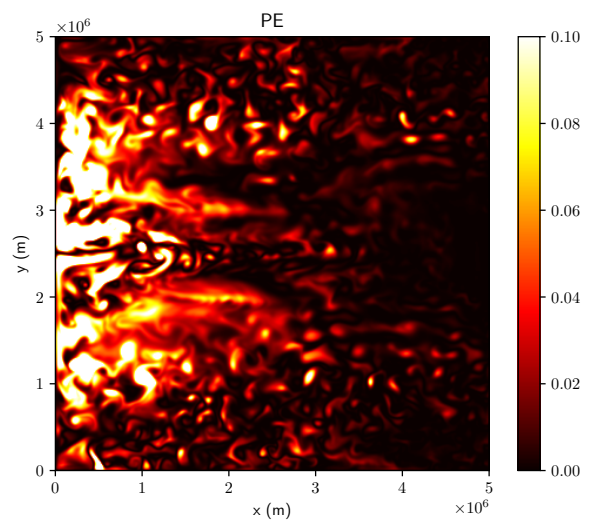

(d) $\overline{P E^{\dagger}}$

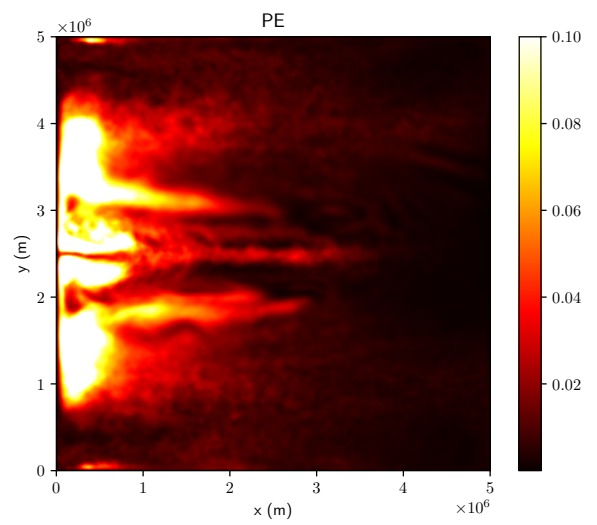

Figure 4. Snapshots and time-mean of potential energy and kinetic energy diagnosed from the eddy model with no forcing $\left(\mathcal{R}^{+}=0\right)$. Units: $\mathrm{m}^{2} \mathrm{~s}^{-2}$

(a) Kinetic energy $[\mathrm{km}]$

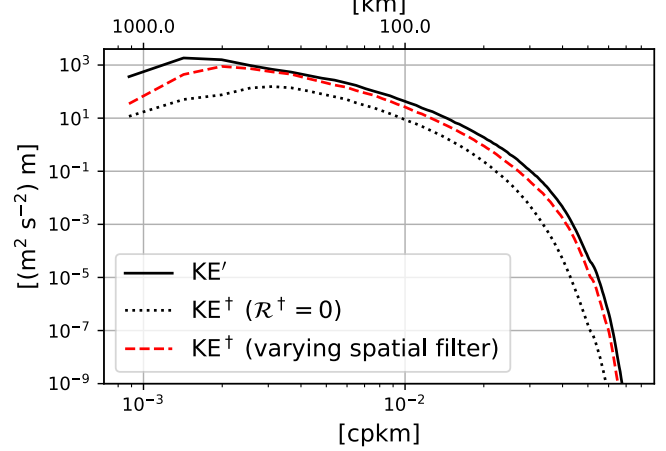

(b) Potential energy

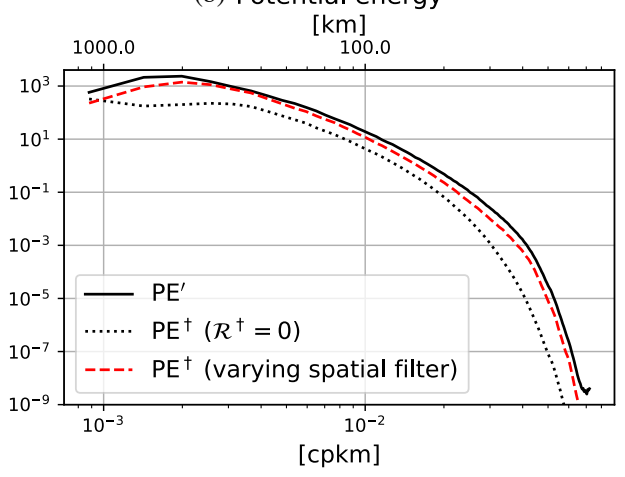

Figure 5. The isotropic wavenumber spectra taken over the whole domain for kinetic and potential energy in the first layer. The energies diagnosed from the full model is shown in solid black, from the eddy model with no forcing in dotted black $\left(\mathcal{R}^{\dagger}=0\right)$, and from the eddy model with the varying spatial filter approach in dashed red lines respectively. 
The interesting point is that in this perturbation run, the large-scale pattern that emerges corresponds to a the cyclonic gyre (in blue) is in the southern part of the domain and the anticyclonic gyre (red) is in the northern part of the domain (Fig. 6b), which is precisely the opposite of the reference run. We interpret this large-scale pattern as the result of the rectification of the large-scale flow by small-scale eddies: the eddies tend to create a flow that opposes the large-scale forcing. As already noted with the energy diagnostics, the intensity of the eddy activity increases near the central latitude and near the western boundary. Near the central latitude, the eddies tend to form an eastward jet, which is also the opposite of what is observed in the reference run (a western boundary current that penetrates into the domain as a westward flowing jet). Although a similar mechanism of the eddies counteracting the mean flow is well known in the Southern Ocean where the overturning circulation by eddies counter balance the mean Ekman steepening of isopycnals [e.g. 46], we conclude that the solution produced by the eddy model $\left(\psi^{\dagger}\right)$ is not a fair reproduction of the eddy dynamics in the full model $\left(\psi^{\prime}\right.$; Fig. 6). We show in section 4 , however, that we have some success in recovering the eddy dynamics from the dagger fields by applying a spatial filter.

(a) $\psi^{\prime}$

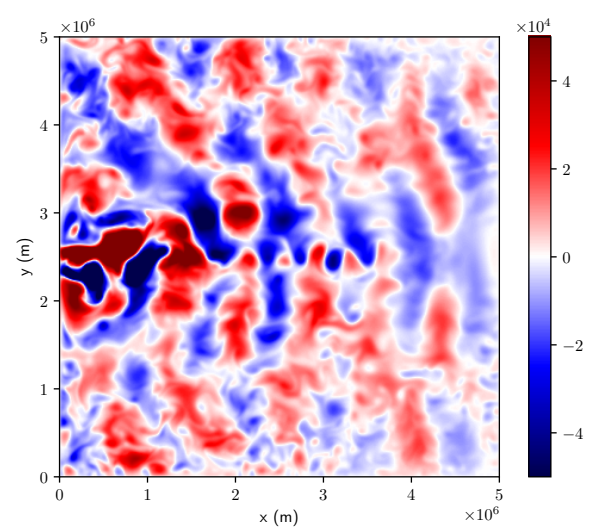

(b) $\psi^{\dagger}$

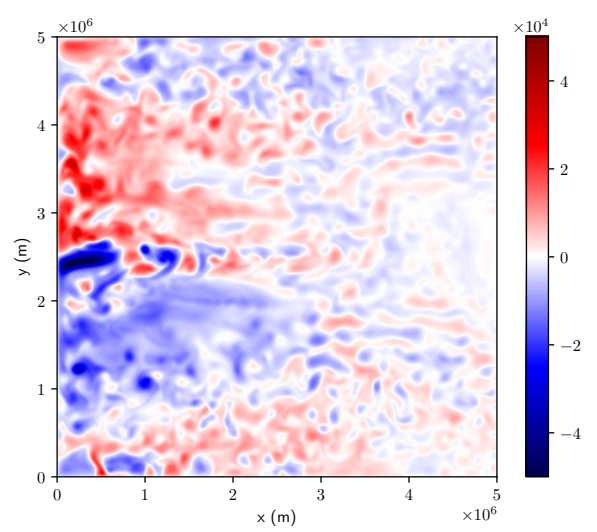

Figure 6. The eddy stream function $\psi^{\prime}$ diagnosed from the full model and eddy stream function $\psi^{\dagger}$ simulated from the eddy model with no forcing $\left(\mathcal{R}^{\dagger}=0\right)$.

We now focus on the rectification term $\overline{J\left(\psi^{\dagger}, q^{\dagger}\right)}$ (the mean of second term on the left-hand side of Eq. 11) that emerges in this simulation from the white-noise initial condition and plot this field in Fig. 7. The field is smoothed in a similar manner to as described in section 2.6 where we average 16 neighboring grid points and linearly interpolate back on the fine grid for visualization purposes. The smoothed $\overline{J\left(\psi^{\dagger}, q^{\dagger}\right)}$ shares many common features with the diagnosed rectification term $\left(\overline{J\left(\psi^{\prime}, q^{\prime}\right)}\right.$; Fig. 2): both fields are positive (negative) in the subpolar (subtropical) gyre. The magnitude of this term is intensified in the region of the separated jet with roughly the same alternance of positive and negative pattern. And last the boundary dynamics is also of the same sign. The main difference is that the simulated field $\overline{J\left(\psi^{\dagger}, q^{\dagger}\right)}$ is weaker in magnitude than the diagnosed field (Fig. 2). The agreement in spatial patterns between these two fields is pleasing given the discrepancies of the dynamics in the two simulations (cf. Figs. 1, 4, 6).

This experiment suggests that eddy dynamics feedback onto the large-scale dynamics via the inverse cascade. In the perturbation model, this feedback on the large-scale potential energy concurs to flatten isopycnal surfaces and effectively shuts off the generation of eddies via baroclinic instability. We conclude that although the term $\overline{J\left(\psi^{\prime}, q^{\prime}\right)}$ has no impact on the domain-integrated energetics of the eddy flow, it is actually very important to counteract the inverse cascade and prevent the formation of spurious large-scale mode in the eddy flow. Even though the stream function itself we get from the eddy 


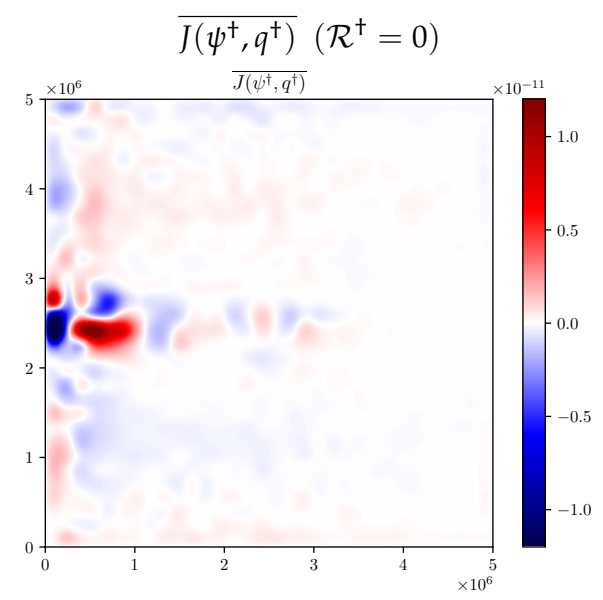

Figure 7. $\overline{J\left(\psi^{\dagger}, q^{\dagger}\right)}$ diagnosed from the eddy model without forcing $\left(\mathcal{R}^{\dagger}=0\right)$, smoothed by averaging 16 neighboring grid points and linearly interpolated back on the fine grid.

model is different from the diagnosed eddy stream function from the full model, we get at this point a viable candidate for the rectification of the large-scale flow by the eddies $\left(\overline{J\left(\psi^{\dagger}, q^{\dagger}\right)}\right)$. In the remainder of this analysis, we propose two strategies to reintroduce this term as a forcing in the eddy equation and examine if we can get a better estimate of the eddy field and the rectification term.

\section{Spatial filtering}

As we we have just described, the solution of the full model exhibits a double gyre pattern that is anticyclonic in the southern part of the domain and cyclonic in the northern part of the domain. Superimposed to this large-scale pattern, we observe an active turbulent activity. Although there is no clear spectral gap between the large-scale circulation and the mesoscale circulation, Pedlosky [47], Grooms et al. [48] and others have proposed to decompose the flow into a large-scale component and a small-scale component. We adopt this strategy and propose to approximate the eddy flow as small scale only. We thus replace the term $\overline{J\left(\psi^{\prime}, q^{\prime}\right)}$ in Eq. (11) by a spatial filter $\mathcal{F}$ whose effect is to damp any large-scale pattern that would emerge from the non-linear interactions in the eddy flow.

\subsection{Scale decomposition}

In order to prevent the formation of a large-scale mode in the eddy equation, we use a spatial filtering approach to mimic the rectification term in Eq. (11). The idea behind this filtering strategy is that even if $\overline{J\left(\psi^{\prime}, q^{\prime}\right)}$ is very slow to converge, we can ensure that the eddy solution remains on the deformation scale. We can already anticipate that this strategy will not work well in the region of the separated jet where there is no clear scale separation between the eddy flow and the mean flow [cf. 49]. However, as we shall see, this strategy works well in the rest of the domain.

We first introduce the scale decomposition for a field $\psi$ as

$$
\psi=\widetilde{\psi}+\psi^{*},
$$

where $\widetilde{\psi}$ and $\psi^{*}$ are respectively the large-scale and small-scale components of the field $\psi$. We do this scale separation by applying a low-pass filter with a discrete wavelet transform (numerical details of the implementation are provided in the Appendix). We illustrate this decomposition in Fig. 8 where we plot the same stream function as the one used in Fig. 4 along with its large-scale and small-scale component. In Fig. 8, we use a cutoff length scale of $\lambda_{c}=500 \mathrm{~km}$. In the large-scale pattern, we recognize a cyclonic and anticyclonic gyre, and a weak jet in the middle that we described earlier. 
(a) $\widetilde{\psi^{\dagger}}$

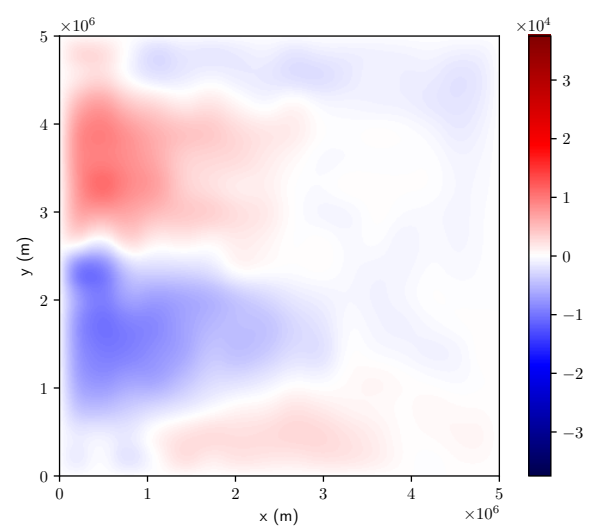

(b) $\psi^{\dagger *}$

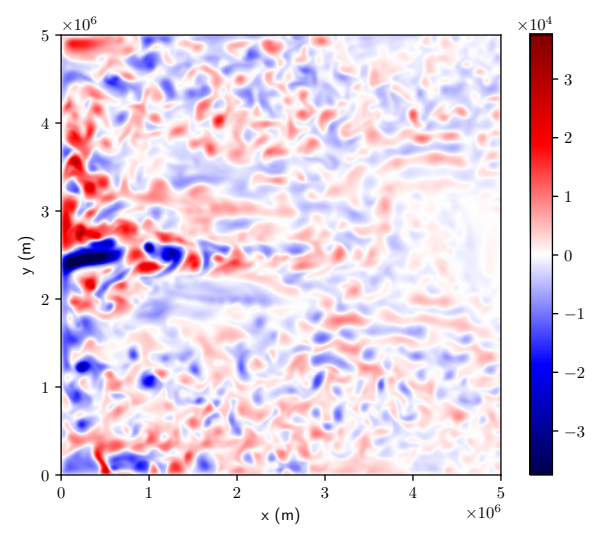

Figure 8. Low pass and high pass filtered eddy stream function diagnosed from the eddy model with no forcing $\left(\mathcal{R}^{+}=0\right)$. The eddy stream function spatially decomposed is the one in Fig. $6 \mathrm{~b}$.

\subsection{Filtering of the large-scale mode in the small scale equation}

Based on Fig. 8b we hypothesize that the eddy rectification term can be approximated by the small scale flow. Namely, we use the scale decomposition to periodically remove the large scale component in Eq. (11) as we see in Fig. 8a. Formally we apply the following operator detailed in the Appendix

$$
\mathcal{F}(\psi)=\psi-\widetilde{\psi}
$$

to the stream function $\psi^{\dagger}$ in Eq. (11) every three days (viz. $\mathcal{F}\left(\psi^{\dagger}\right)=\psi^{t^{*}}$ ). We choose this three-day period because it is comparable to the eddy time scale and is short enough compared to the time needed to build the large-scale mode observed in Fig. 8a which is on the order of years. In order to facilitate the following discussion, we re-write the eddy model (Eq. 11) using the prognostic dagger variables

$$
\frac{\partial q^{\dagger}}{\partial t}+J\left(\psi^{\dagger}, q^{\dagger}\right)+J\left(\bar{\psi}, q^{\dagger}\right)+J\left(\psi^{\dagger}, \bar{q}\right)+\beta v^{\dagger}=A_{4} \nabla^{4} q^{\dagger}+r_{b} \nabla^{2} \psi^{\dagger}+\mathcal{R}^{\dagger},
$$

where we represent the eddy rectification forcing with $\mathcal{R}^{\dagger}=\mathcal{T}^{-1} \mathcal{L}\left[\mathcal{F}\left(\psi^{\dagger}\right)\right] . \mathcal{L}$ is the linear operator in Eq. (1), and $\mathcal{T}$ is the three-day time scale. The time scale separation is similar to ocean models where the barotropic and baroclinic modes are solved with different time stepping [cf. 50]. The time scale separation can be rephrased as we are enforcing

$$
\frac{\partial \tilde{q}^{\dagger}}{\partial t}=0
$$

with the initial condition of $\tilde{q^{\dagger}}(t=0)=0$ so that $\tilde{q}^{\dagger}=0 a n d q^{\dagger}=q^{t^{*}}$ is satisfied for all time.

For this first experiment, we choose the filter cutoff length as $\lambda_{c}=1000 \mathrm{~km}$ which corresponds to roughly 4 times the average instability length scale and is thus in between the eddy scale and the basin scale. We plot the energy diagnostic of this run in Fig. 9 using the same layout as in Fig. 4. These energy diagnostics exhibit different features than the previous eddy run. The most striking feature is that there is more kinetic energy and less potential energy everywhere in the domain. Eddies are now more abundant in the basin: not only in the region of the separated jet but also in the return flow of both gyres. Also the jet at mid latitude now flows from west to east; this is in the same direction as the mean flow. As expected, the use of the filters removes the large-scale component of the flow such that the spurious large-scale pattern that where visible in Fig. $4 \mathrm{~b}$ are no longer visible in Fig. 9b. Comparing Fig. 9 with Figs. 1 and 4, we see that 
there is a clear improvement in extracting the eddy dynamics using the spatial filter with similar westward penetration of the separated jet into the basin. In the region of the separated jet, the the eddy flow $\left(\mathcal{F}\left(\psi^{\dagger}\right)\right)$ still underestimates the magnitude of eddy activity $\left(\psi^{\prime}\right)$.

(a) $K E^{\dagger}$

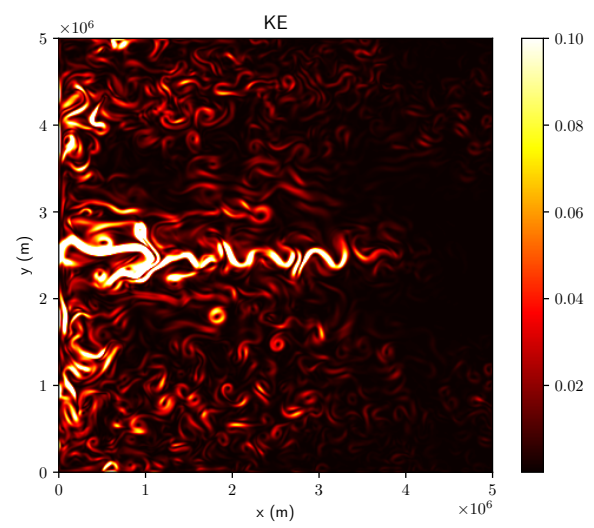

(c) $\overline{K E^{\dagger}}$

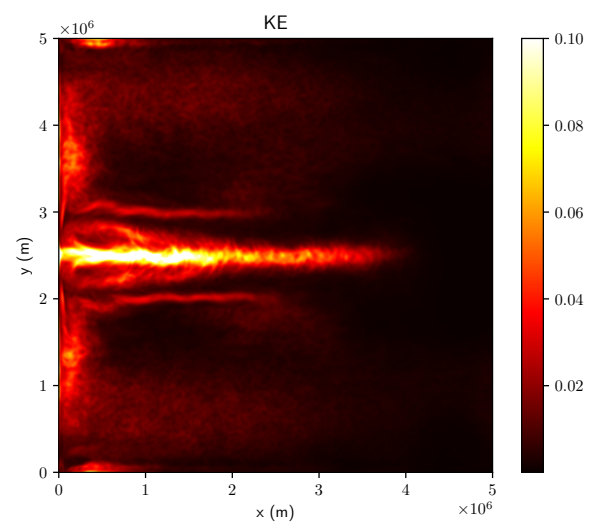

(b) $P E^{\dagger}$

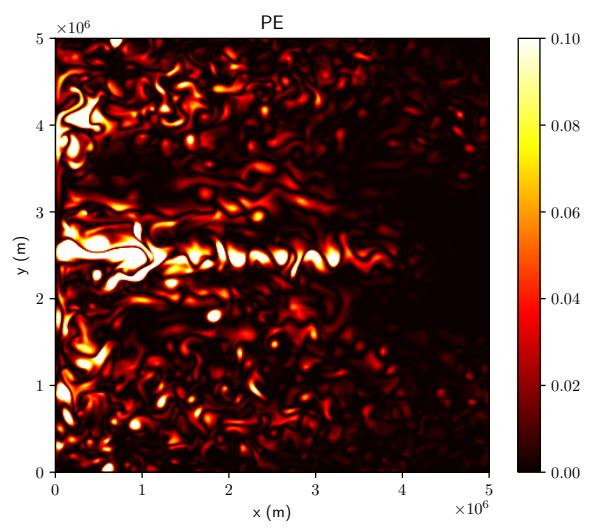

(d) $\overline{P E^{\dagger}}$

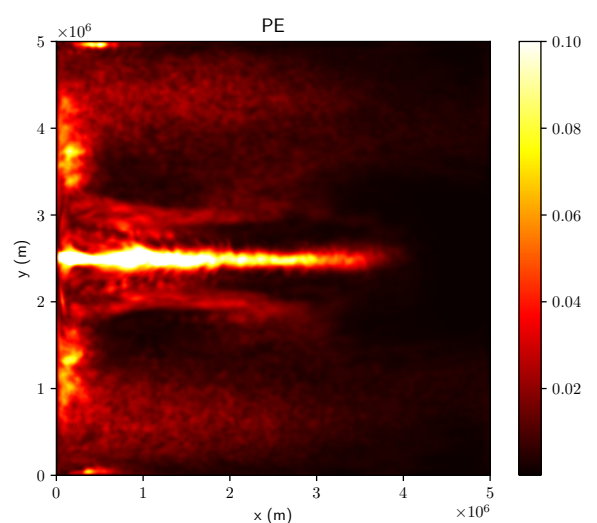

Figure 9. Snapshots and time-mean potential energy and kinetic energy diagnosed from the eddy model where $\mathcal{R}^{\dagger}$ is implemented with a spatially-uniform scale filter. Units: $\mathrm{m}^{2} \mathrm{~s}^{-2}$

We performed several runs with different values of $\lambda_{c}$ and find that when $\lambda_{c}$ is greater than $1000 \mathrm{~km}$ we recover the solution with no filter. For smaller values of $\lambda_{c}$ we observe a nearly uniform eddy field for which the size of the eddies is of the order of $\lambda_{c}$ (not shown).

\subsection{Variable length scale filter}

We now allow the length scale of the spatial filter to vary in space. It is clear from the previous simulation that the energy level in the eddy field $\left(\left|\nabla \mathcal{F}\left(\psi^{\dagger}\right)\right|^{2}\right)$ is still smaller than in the original simulation. In fact, we see in Fig. 1a that the patch of high eddy kinetic energy has horizontal dimensions on the order of $1000 \mathrm{~km}$, which is precisely the cutoff length scale of the filter. In the region of the separated jet, there is thus no clear scale separation between the eddy flow and the mean flow. To a certain extent, this corroborates what we observed in the instability analysis. In Fig. 3, we see that in the region of the separated jet, the most unstable mode has $\lambda=300 \mathrm{~km}$ compared to $\lambda=230 \mathrm{~km}$ in the return flow. We use that information to build a filter with non uniform length scale in the form of $\lambda_{c}=\alpha \lambda$. If we set $\alpha=4.5$, we get $\lambda_{c} \sim O(1000 \mathrm{~km})$ in the area of the return flow, and we have just found above that this value gives correct results in most of the gyre. With the combination of $\alpha=4.5$ and $\lambda=300 \mathrm{~km}$, we would get $\lambda_{c} \simeq 1350 \mathrm{~km}$. However, using the raw value of $\lambda$ as shown in Fig. $3 \mathrm{~b}$ with $\alpha=4.5$ is 
not necessarily a good idea because this field is noisy and also because some instabilities are not relevant to the dynamics. This occurs in places where the instability time scale is greater than the advection time scale (which is on the order of 20 days in most of the gyre, not shown). To get rid of the non relevant unstable modes, we adjust the value of $\lambda$ to $225 \mathrm{~km}$ everywhere where $\tau>115$ days. We then smooth that field to get rid of the grid scale variations. Lastly, for each point of the domain, we create a halo of size $\alpha \lambda$ over which we propagate the value of $\lambda$. Several halos overlap at one point and so for each point we retain the maximum value of all halos that are present at that point. This is done to let enough space for all instabilities to develop around the formation site. We smooth the final map to damp the halo pattern that may have persisted. We plot the final map of $\lambda_{c}$ in Fig. 10. As desired, $\lambda_{c}$ has values on the order of $1000 \mathrm{~km}$ with a maximum of $1350 \mathrm{~km}$ in the region of the separated jet and a minimum of $850 \mathrm{~km}$ near the north-east and south-east corners.

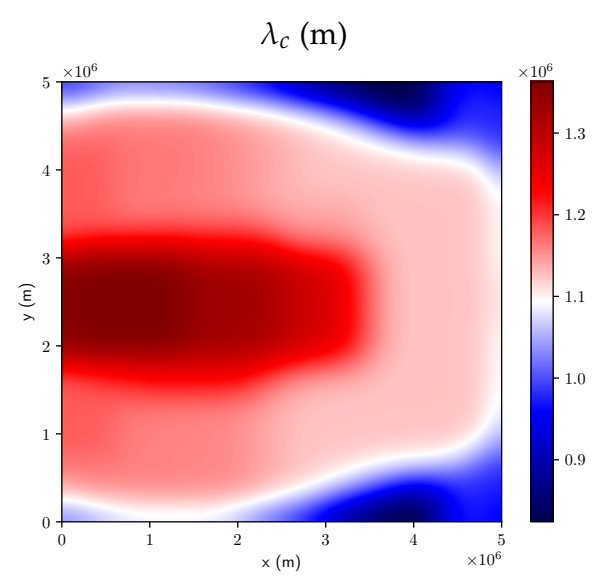

Figure 10. The cut-off length scale $\left(\lambda_{c}\right)$ based on the instability length scale.

We plot the energy diagnostics of the variable length scale filter in Fig. 11. Comparing Fig. 11c with $9 c$, and $11 d$ with $9 d$, we see that the varying filter size succeeds in increasing the eddy amplitude overall and in particular around the separated jet. The energy levels come closer to the eddy field diagnosed from the full model (Figs. 1c and 1d), which is also apparent in the isotropic wavenumber spectra (Fig. 5). We see clear increase in energy from the run without forcing and that the varying spatial filter approach captures energy levels close to the diagnosed eddy kinetic and potential energy except for the smallest wavenumbers (largest spatial scales; compare the black solid and red dashed lines in Fig. 5). This is expected as we remove the large-scale component with the spatial filter $\mathcal{F}$.

If we average Eq. (21), the terms linear in dagger vanish and one should get a balance between

$$
\overline{J\left(\psi^{\dagger}, q^{\dagger}\right)} \simeq \overline{\mathcal{R}^{\dagger}}
$$

Although the balance in Eq. (23) requires there to be a clear scale separation between the eddy and mean flow, we expect this to approximately hold, viz. $\overline{\psi^{\dagger}} \sim 0$ for a converged simulation. Equation (23) is complimentary to a recent work by Porta Mana and Zanna [31] and Grooms and Zanna [51] where they find a local relation $\overline{J\left(\psi^{\prime s}, q^{\prime s}\right)^{s}} \simeq \nabla^{2} \frac{D \bar{q}^{s}}{D t}$ and $\overline{(\cdot)^{s}}$ is their spatially filtered field and $(\cdot)^{\prime s}\left(=(\cdot)-\overline{(\cdot)^{s}}\right)$ the residual from their filtered field. One may question why go through the hassle of solving Eq. (21) when you can already have an estimate for the eddy rectification term (Eq. 23). We emphasize that by explicitly solving for Eq. (21) and diagnosing the eddy rectification term from it, the rectification term incorporates non-local effects.

We plot in Fig. 12, $\overline{J\left(\psi^{\dagger}, q^{\dagger}\right)}$ and $\overline{\mathcal{R}^{\dagger}}$. (The difference between Fig. 12a and 7 is in Eq. (21) prognostically solved with and without the eddy rectification forcing on the right-hand side.) We first see that $\overline{J\left(\psi^{\dagger}, q^{\dagger}\right)}$ captures the same patterns as the diagnosed 
(a) $K E^{+}$

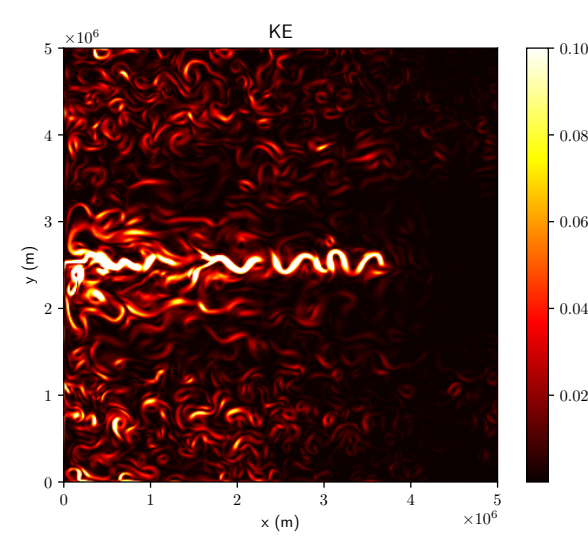

(c) $\overline{K E^{\dagger}}$

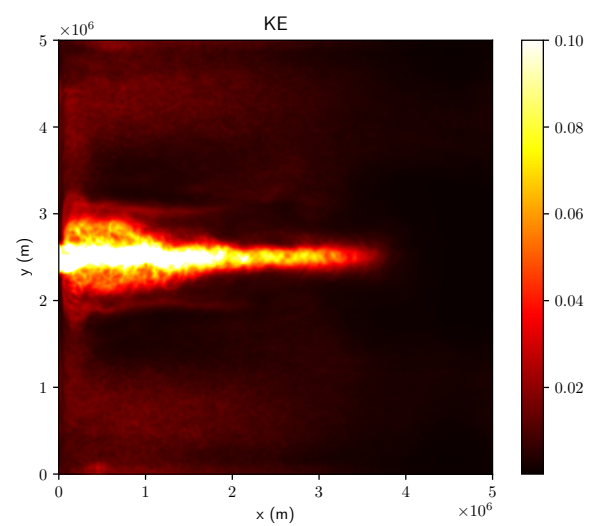

(b) $P E^{\dagger}$

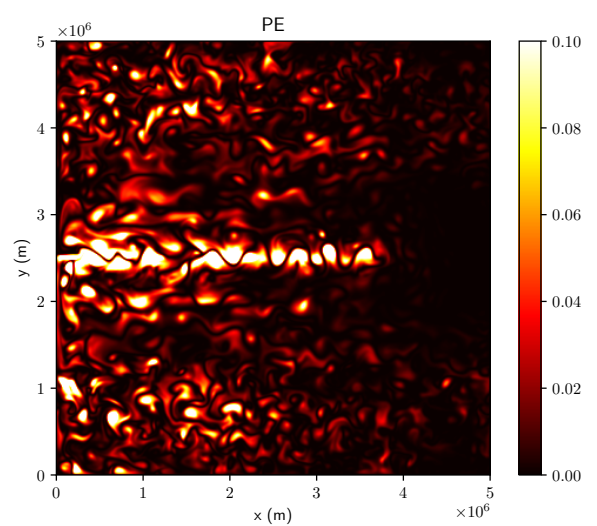

(d) $\overline{P E^{\dagger}}$

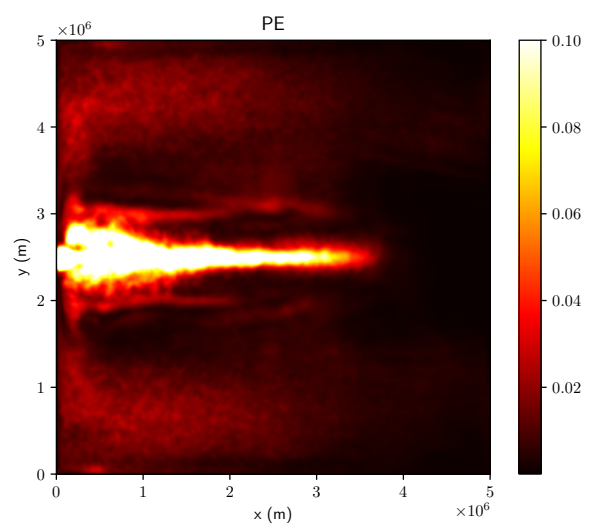

Figure 11. Potential energy and kinetic energy diagnosed from the eddy model where $\mathcal{R}^{\dagger}$ is implemented with the variable length scale filter. Units: $\mathrm{m}^{2}$ 
field from the full model $\overline{J\left(\psi^{\prime}, q^{\prime}\right)}$. We see improvements compared to the run without the rectification forcing $\left(\mathcal{R}^{\dagger}=0\right.$; Fig. 13); the joint histogram of $\overline{J\left(\psi^{\prime}, q^{\prime}\right)}$ and $\overline{J\left(\psi^{\dagger}, q^{\dagger}\right)}$ aligns more around the one-to-one line with the varying spatial filter approach. This is an important result because it means that one can use this model (Eq. 21) to reproduce the eddy statistics. If we now compare $\overline{J\left(\psi^{\dagger}, q^{\dagger}\right)}$ and $\overline{\mathcal{R}^{\dagger}}$, we see that $\overline{\mathcal{R}^{\dagger}}$ captures the large scale pattern in the return flow of the gyre but misses the small scale variability in the separated jet and right at the western boundary. We could have anticipated this result because of the nature of our filter which leaves small scale dynamics unchanged and slow convergence of the eddy field as we discussed in section 2.6. In the separated jet, we face here the limits of our approximation of the time average by a low-pass filter. We also observe that reducing the length scale of the filter is problematic because it degrades the quality of the eddy solution. Nevertheless, even with this bias, the rectification term $\left(\overline{J\left(\psi^{\dagger}, q^{\dagger}\right)}\right)$ compares well with the diagnosed rectification $\left(\overline{J\left(\psi^{\prime}, q^{\prime}\right)}\right.$; Figs. 2 and 12).

(a) $\overline{J\left(\psi^{\dagger}, q^{\dagger}\right)}$ (varying spatial filter)

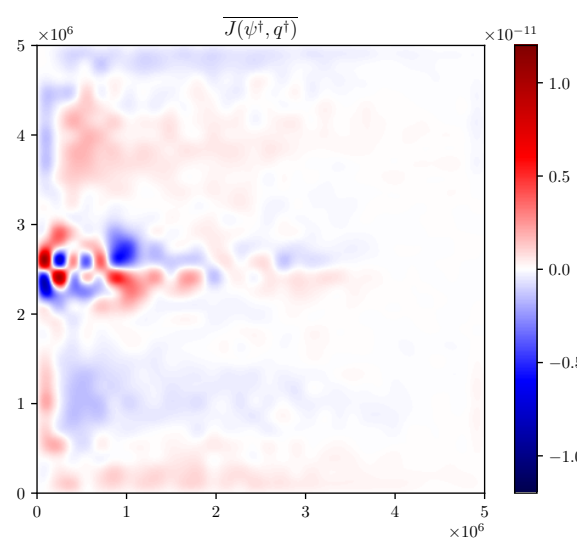

(b) $\overline{\mathcal{R}^{\dagger}}$

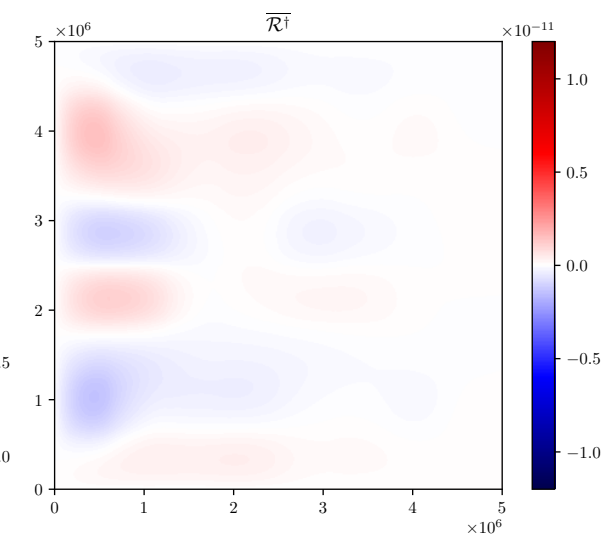

Figure 12. $\overline{J\left(\psi^{\dagger}, q^{\dagger}\right)}$ diagnosed from the eddy model with the varying spatial filter approach, smoothed by averaging 16 neighboring grid points and linearly interpolated back on the fine grid, and $\overline{\mathcal{R}^{\dagger}}$.

\section{Conclusions and discussion}

In this study, we have examined the eddy rectification term, which encapsulates the net eddy feedback onto the mean flow, from a quasi-geostrophic (QG) double gyre simulation. In doing so, we decompose the QG potential vorticity (PV) into its mean flow, defined by a time mean, and eddies as the fluctuations about the mean. The eddy rectification term is then diagnosed from the full model (Eq. 10) and eddy model (Eq. 11). We have shown that the unforced eddy model $\left(\mathcal{R}^{\dagger}=0\right)$ gives a rough estimate for the rectification term diagnosed from the full model, viz. $\overline{J\left(\psi^{\dagger}, q^{\dagger}\right)} \sim \overline{J\left(\psi^{\prime}, q^{\prime}\right)}$ (Figs. $2 \mathrm{~b}$ and 7). However, the fact that a large-scale component of the eddy stream function itself $\left(\tilde{\psi}^{\dagger}\right)$ emerges opposing the mean flow without the eddy rectification forcing, which is not apparent in the eddy stream function diagnosed from the full model $\left(\psi^{\prime}\right)$, perhaps warrants some attention (Figs. 6 and 8). Previous studies have solved the eddy model without the forcing [e.g. 28,29]. This has partially been due to the fact that the eddy rectification term is difficult to accurately diagnose. We have shown that approximating the eddy rectification forcing by the spatially-filtered eddy stream function $\left(\mathcal{R}^{\dagger} \simeq \mathcal{T}^{-1} \mathcal{L}\left[\mathcal{F}\left(\psi^{\dagger}\right)\right]\right)$ improves the eddy kinetic and potential energy and $\overline{J\left(\psi^{\dagger}, q^{\dagger}\right)}$ (Figs. 5, 11-13).

In the context of parametrizing the eddy feedback onto the mean flow, we have shown a spatial filtering approach. Once the eddy rectification terms, which are enforced to be dynamically consistent with the mean flow, are diagnosed from the eddy 
(a)

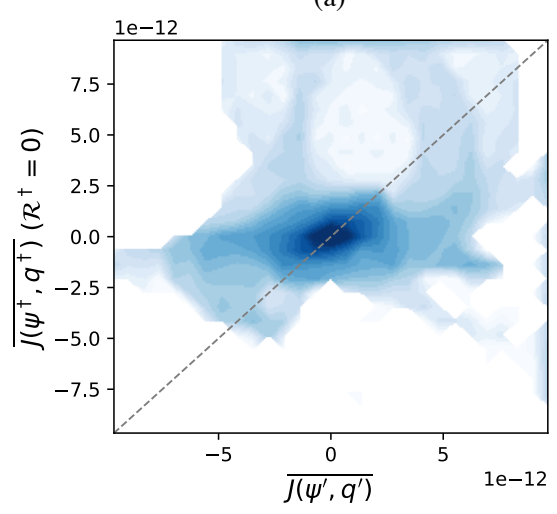

(c)

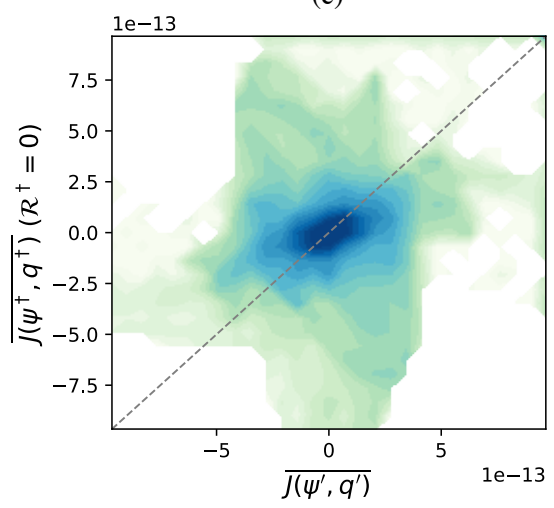

First layer

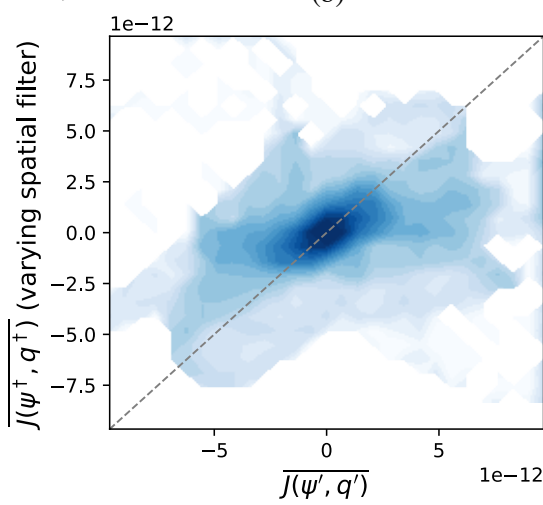

(d)

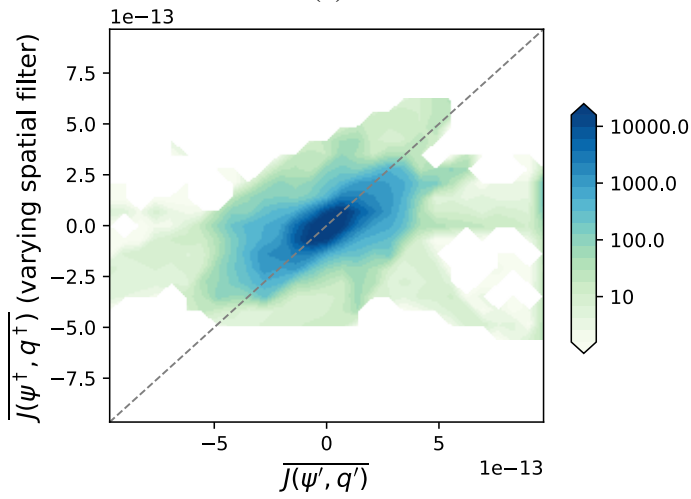

Figure 13. Joint histogram of the spatially smoothed $\overline{J\left(\psi^{\prime}, q^{\prime}\right)}$ and $\overline{J\left(\psi^{\dagger}, q^{\dagger}\right)}$ for the first and second layer plotted against a logarithmic scaling (the masked out regions have zero values). The left column shows the run with no forcing $\left(\mathcal{R}^{\dagger}=0\right)$ and right the run with the varying spatial filter approach. The one-to-one line is shown in grey dashed lines. The histograms were computed using the xhistogram Python package [52]. 
model $\left(\overline{J\left(\psi^{\dagger}, q^{\dagger}\right)}\right.$; Eq. 21$)$, we can then plug them back into the mean flow model (Eq. 10 ) as a forcing term on the right-hand side. This is similar to other energy backscatter parametrization studies where they solve the (unresolved) eddy energy equation and take that as an input for the resolved energy equation [e.g. 23,25,26]. Here, we have formulated a deterministic closure based on PV instead of energy; PV is a more fundamental variable in quasi geostrophy as momentum is invertible from it. Our approach of parametrizing the eddy rectification term via a spatially-filtered eddy stream function $\left(\mathcal{F}\left(\psi^{\dagger}\right)\right)$ is complementary to a recent work by Mana and Zanna [38] and Grooms and Zanna [51] where they find a closure for the rectification term in relation to the low-pass filtered PV. One major difference here is that while their closure was local, we have accounted for non-local effects by solving the eddy model prognostically. We are also currently looking into stochastic closures.

Other than the spatial filtering approach, it is theoretically possible to obtain the rectification term through iteratively solving for Eq. (21) as the Fixed-Point Theorem would predict. As we discussed in section 3.2, the eddy model without any forcing term $\left(\mathcal{R}^{\dagger}=0\right)$ produces a good first guess of the rectification term, namely the mean of $J\left(\psi^{\dagger}, q^{\dagger}\right)$ on the left-hand side of Eq. (21) (Fig. 7). The idea is then to re-run the eddy model with this first guess as the forcing term $\left(\mathcal{R}^{\dagger}=\overline{J\left(\psi^{\dagger}, q^{\dagger}\right)}\right)$ and repeat this iterative procedure until convergence is reached. We already know that this convergence is extremely slow (order of million of eddy time scale; section 2.6) so this process can not be practically done with the raw estimate of the rectification term but may be possible for its spatially smoothed version. The proof for mathematical convergence of this iterative process is beyond the scope of this study and will be left for interested mathematicians.

Another notable point is that because we solve for the mean and eddy model prognostically, our closure applies for the total PV $\left(q=\bar{q}+q^{\dagger}\right.$, and stream function $\psi=\mathcal{L}^{-1} q$ ) at each time step as opposed to solely the mean PV. Commonly, the approach for mesoscale closures has been to focus on the mean equations including recent developments in energy backscattering parametrizations [e.g. 6,22-27]. We argue that it is actually more beneficial to develop a closure which couples the mean and eddy model, as we have attempted here, to capture the total variability otherwise resolved under sufficient model resolution. For realistic simulations, in addition to the mean state, we are often interested in fluctuations about the mean and extreme events [e.g. 53-55]; having a closure for the total PV accounts for both in a physically consistent manner. Such approach is sometimes referred to as super parametrizations and have been commonly implemented for atmospheric convection [e.g. 56,57]. Lastly, one may ask how our results can be extended to primitive equation models. In primitive equations, the eddy Ertel PV flux encapsulates the eddy feedback onto the mean flow [58]. In other words, a closure based on Ertel PV may allow one to capture the net eddy-mean flow interaction and variability in the total Ertel PV. We leave this for future work.

Author Contributions: Conceptualization, B.D.; methodology, B.D.; software, B.D. \& S.P.; validation, B.D.; formal analysis, B.D. \& T.U.; investigation, B.D. \& T.U.; resources, B.D.; data curation, B.D.; writing, B.D. \& T.U.; visualization, B.D. \& T.U.; project administration, B.D.; funding acquisition, B.D. \& T.U. All authors have read and agreed to the published version of the manuscript.

Funding: This work has been supported by the French national programme LEFE/INSU. T.U. acknowledges support from the French 'Make Our Planet Great Again' (MOPGA) initiative managed by the Agence Nationale de la Recherche under the Programme d'Investissement d'Avenir, with the reference ANR-18-MPGA-0002.

Data Availability Statement: The open-source software for the QG model used can be found on Github [35]. For simulation outputs, please contact the corresponding author.

Acknowledgments: We wish to thank Antoine Venaille, Laure Saint Raymond and William K. Dewar for their insightful comments and suggestions.

Conflicts of Interest: The authors declare no conflict of interest. 


\section{Appendix A}

Appendix A.1 Numerical implementation of the spatial filter

The discrete wavelet transform bears some resemblance with the multigrid solver. We define a set of grids from the finest model resolution $2^{n} \times 2^{n}$ to the coarsest resolution $2^{0} \times 2^{0}$ (one grid point). In our model, there are $n+1=10$ sets of grids. The two key operations in the filtering procedure are

- $\quad$ The restriction $\mathcal{R}$ for which we coarsen a field by averaging 4 neighboring points

- The prolongation $\mathcal{P}$ for wich we refine a field by linear interpolation of neighboring points.

Let suppose a field $\psi^{l}$ is defined on a grid $2^{l} \times 2^{l}$. We say it is defined of a grid of level $l$ for which the grid step is $\Delta_{l}=L / 2^{l}$. Then we have

$$
\psi^{l-1}=\mathcal{R}\left(\psi^{l}\right),
$$

We define the wavelet coefficients at level $l$ as

$$
\check{\psi}^{l}=\psi^{l}-\mathcal{P}\left(\psi^{l-1}\right) .
$$

Hence from the wavelet coefficients, one can reconstruct the field at the finest grid with an iterative procedure. The wavelet coefficients at level $l$ hold the information about the structure of the field at length scale of the grid size $\Delta_{l}$. To high pass filter a field with a cutoff length scale $\lambda_{c}=\Delta_{k}$, we simply need to set to zero the wavelet coefficients $\breve{\psi}^{l}$ for $l<k$. In case $\lambda_{c}$ varies smoothly in space we can zero the wavelet coefficients locally only.

\section{References}

1. Smagorinsky, J. General Circulation Experiments with the Primitive Equations. Mon. Wea. Rev. 1963, 91, 99-164. doi:10.1175/15200493(1963)091<0099:GCEWTP>2.3.CO;2.

2. Launder, B.E.; Reece, G.J.; Rodi, W. Progress in the development of a Reynolds-stress turbulence closure. J. Fluid Mech. 1975, 68, 537-566. doi:10.1017/S0022112075001814.

3. Large, W.G.; McWilliams, J.C.; Doney, S.C. Oceanic vertical mixing: a review and a model with a nonlocal boundary layer parameterization. Rev. Geophys. 1994, 32, 363-404. doi:10.1029/94RG01872.

4. Mellor, G.L.; Yamada, T. Development of a turbulence closure model for geophysical fluid problems. Rev. Geophys. 1982, 20, 851-875. doi:10.1029/RG020i004p00851.

5. Redi, M.H. Oceanic isopycnal mixing by coordinate rotation. J. Phys. Oceanogr. 1982, 12, 1154-1158. doi:10.1175/15200485(1982)012<1154:OIMBCR>2.0.CO;2.

6. Gent, P.R.; McWilliams, J.C. Isopycnal Mixing in Ocean Circulation Models. J. Phys. Oceanogr. 1990, 20, 150-160. doi:10.1175/15200485(1990)020<0150:IMIOCM>2.0.CO;2.

7. Bachman, S.D.; Fox-Kemper, B.; Pearson, B. A scale-aware subgrid model for quasi-geostrophic turbulence. J. Geophys. Res.: Oceans 2017, 122, 1529-1554. doi:10.1002/2016JC012265.

8. Vallis, G.K. Atmospheric and Oceanic Fluid Dynamics; Cambridge University Press, 2006; p. 745.

9. Arbic, B.K.; Polzin, K.L.; Scott, R.B.; Richman, J.G.; Shriver, J.F. On eddy viscosity, energy cascades, and the horizontal resolution of gridded satellite altimeter products. J. Phys. Oceanogr. 2013, 43, 283-300. doi:10.1175/JPO-D-11-0240.1.

10. Aluie, H.; Hecht, M.; Vallis, G.K. Mapping the Energy Cascade in the North Atlantic Ocean: The Coarse-Graining Approach. J. Phys. Oceanogr. 2018, 48, 225-244. doi:10.1175/JPO-D-17-0100.1.

11. Stammer, D. Global characteristics of ocean variability estimated from regional TOPEX/POSEIDON altimeter measurements. $J$. Phys. Oceanogr. 1997, 27, 1743-1769. doi:10.1175/1520-0485(1997)027<1743:GCOOVE>2.0.CO;2.

12. Xu, Y.; Fu, L.L. Global variability of the wavenumber spectrum of oceanic mesoscale turbulence. J. Phys. Oceanogr. 2011, 41, 802-809. doi:10.1175/2010JPO4558.1.

13. Uchida, T.; Abernathey, R.; Smith, S. Seasonality of eddy kinetic energy in an eddy permitting global climate model. Ocean Model. 2017, 118, 41-58. doi:10.1016/j.ocemod.2017.08.006.

14. Ajayi, A.; Le Sommer, J.; Chassignet, E.; Molines, J.M.; Xu, X.; Albert, A.; Cosme, E. Spatial and Temporal Variability of the North Atlantic Eddy Field From Two Kilometric-Resolution Ocean Models. J. Geophys. Res.: Oceans 2020, 125, e2019JC015827. doi:10.1029/2019JC015827.

15. Chassignet, E.P.; Xu, X. Impact of Horizontal Resolution $\left(1 / 12^{\circ}\right.$ to $\left.1 / 50^{\circ}\right)$ on Gulf Stream Separation, Penetration, and Variability. J. Phys. Oceanogr. 2017, 47, 1999-2021. doi:10.1175/JPO-D-17-0031.1. 
16. Kjellsson, J.; Zanna, L. The impact of horizontal resolution on energy transfers in global ocean models. Fluids $2017,2,45$. doi:10.3390/fluids2030045.

17. Kelly, K.; Small, R.; Samelson, R.; Qiu, B.; Joyce, T.; Kwon, Y.O.; Cronin, M. Western Boundary Currents and Frontal Air-Sea Interaction: Gulf Stream and Kuroshio Extension. J. Climate 2010, 23, 5644-5667. doi:10.1175/2010JCLI3346.1.

18. Tréguier, A.M.; Deshayes, J.; Le Sommer, J.; Lique, C.; Madec, G.; Penduff, T.; Molines, J.M.; Barnier, B.; Bourdallé-Badie, R.; Talandier, C. Meridional transport of salt in the global ocean from an eddy-resolving model. Ocean Science 2014, 10, $243-255$. doi:10.5194/os-10-243-2014.

19. Jones, C.; Cessi, P. Components of Upper-Ocean Salt Transport by the Gyres and the Meridional Overturning Circulation. J. Phys. Oceanogr. 2018, 48, 2445-2456. doi:10.1175/JPO-D-18-0005.1.

20. Bellucci, A.; Athanasiadis, P.; Scoccimarro, E.; Ruggieri, P.; Gualdi, S.; Fedele, G.; Haarsma, R.; Garcia-Serrano, J.; Castrillo, M.; Putrahasan, D.; others. Air-Sea interaction over the Gulf Stream in an ensemble of HighResMIP present climate simulations. Climate Dyn. 2020, pp. 1-19. doi:10.1007/s00382-020-05573-z.

21. Zanna, L.; Mana, P.P.; Anstey, J.; David, T.; Bolton, T. Scale-aware deterministic and stochastic parametrizations of eddy-mean flow interaction. Ocean Model. 2017, 111, 66-80. doi:10.1016/j.ocemod.2017.01.004.

22. Berloff, P. Dynamically consistent parameterization of mesoscale eddies. Part III: Deterministic approach. Ocean Model. 2018, 127, 1-15. doi:10.1016/j.ocemod.2018.04.009.

23. Jansen, M.F.; Adcroft, A.; Khani, S.; Kong, H. Toward an energetically consistent, resolution aware parameterization of ocean mesoscale eddies. J. Adv. Model. Earth Syst. 2019, 11, 2844-2860. doi:10.1029/2019MS001750.

24. Bachman, S.D. The GM+ E closure: A framework for coupling backscatter with the Gent and McWilliams parameterization. Ocean Model. 2019, 136, 85-106. doi:10.1016/j.ocemod.2019.02.006.

25. Juricke, S.; Danilov, S.; Kutsenko, A.; Oliver, M. Ocean kinetic energy backscatter parametrizations on unstructured grids: Impact on mesoscale turbulence in a channel. Ocean Model. 2019, 138, 51-67. doi:10.1016/j.ocemod.2019.03.009.

26. Perezhogin, P. Deterministic and stochastic parameterizations of kinetic energy backscatter in the NEMO ocean model in Double-Gyre configuration. IOP Conf. Series: Earth and Environ. Sci. IOP Publishing, 2019, Vol. 386, p. 012025. doi:10.1088/1755$1315 / 386 / 1 / 012025$.

27. Zanna, L.; Bolton, T. Data-Driven Equation Discovery of Ocean Mesoscale Closures. Geophys. Res. Lett. 2020, 47, e2020GL088376. doi:10.1029/2020GL088376.

28. Venaille, A.; Vallis, G.K.; Smith, K.S. Baroclinic Turbulence in the Ocean: Analysis with Primitive Equation and Quasigeostrophic Simulations. J. Phys. Oceanogr. 2011, 41, 1605-1623. doi:10.1175/JPO-D-10-05021.1.

29. Grooms, I.; Nadeau, L.P.; Smith, K.S. Mesoscale Eddy Energy Locality in an Idealized Ocean Model. J. Phys. Oceanogr. 2013, 43, 1911-1923. doi:10.1175/JPO-D-13-036.1.

30. Marshall, D.P.; Maddison, J.R.; Berloff, P.S. A framework for parameterizing eddy potential vorticity fluxes. J. Phys. Oceanogr. 2012, 42, 539-557. doi:10.1175/JPO-D-11-048.1.

31. Porta Mana, P.; Zanna, L. Toward a stochastic parameterization of ocean mesoscale eddies. Ocean Model. 2014, 79, 1-20. doi:10.1016/j.ocemod.2014.04.002.

32. Mak, J.; Maddison, J.R.; Marshall, D.P. A new gauge-invariant method for diagnosing eddy diffusivities. Ocean Model. 2016, 104, 252-268. doi:10.1016/j.ocemod.2016.06.006.

33. Berloff, P. Dynamically consistent parameterization of mesoscale eddies. Part I: Simple model. Ocean Model. 2015, 87, 1-19. doi:10.1016/j.ocemod.2014.12.008.

34. Popinet, S. A quadtree-adaptive multigrid solver for the Serre-Green-Naghdi equations. J. Comput. Phys. 2015, 302, 336-358. doi:10.1016/j.jcp.2015.09.009.

35. Deremble, B.; Martinez, E.M. MSOM: Multiple Scale Ocean Model, 2020. doi:10.5281/zenodo.4669909.

36. Josey, S.A.; Kent, E.C.; Taylor, P.K. Wind Stress Forcing of the Ocean in the SOC Climatology: Comparisons with the NCEP NCAR, ECMWF, UWM/COADS, and Hellerman and Rosenstein Datasets. J. Phys. Oceanogr. 2002, 32, 1993. doi:10.1175/15200485(2002)032<1993:WSFOTO>2.0.CO;2.

37. Eden, C. Parameterising meso-scale eddy momentum fluxes based on potential vorticity mixing and a gauge term. Ocean Model. 2010, 32, 58-71. doi:10.1016/j.ocemod.2009.10.008.

38. Mana, P.P.; Zanna, L. Toward a stochastic parameterization of ocean mesoscale eddies. Ocean Model. 2014, 79, 1-20. doi:10.1016/j.ocemod.2014.04.002.

39. Kondrashov, D.; Berloff, P. Stochastic modeling of decadal variability in ocean gyres. Geophysical Research Letters 2015, 42, 15431553. doi:10.1002/2014GL062871.

40. Smith, K.S. The geography of linear baroclinic instability in Earth's oceans. J. Mar. Res 2007, 65, 655-683. doi:10.1357/002224007783649484.

41. Tulloch, R.; Marshall, J.; Hill, C.; Smith, K.S. Scales, Growth Rates, and Spectral Fluxes of Baroclinic Instability in the Ocean. J. Phys. Oceanogr. 2011, 41, 1057-1076. doi:10.1175/2011JPO4404.1.

42. Cushman-Roisin, B.; Beckers, J.M. Introduction to geophysical fluid dynamics: physical and numerical aspects; Vol. 101, Academic Press, 2011.

43. Uchida, T.; Rokem, A.; Squire, D.; Nicholas, T.; Abernathey, R.; Nouguier, F.; Constantinou, N.; others. xrft: Fourier transforms for xarray data, 2021. doi:10.5281/zenodo.4275915. 
44. Khatri, H.; Sukhatme, J.; Kumar, A.; Verma, M.K. Surface ocean enstrophy, kinetic energy fluxes, and spectra from satellite altimetry. J. Geophys. Res.: Oceans 2018, 123, 3875-3892. doi:10.1029/2017JC013516.

45. Charney, J.G. Geostrophic Turbulence. J. Atmos. Sci. 1971, 28, 1087-1094. doi:10.1175/1520-0469(1971)028<1087:GT>2.0.CO;2.

46. Sinha, A.; Abernathey, R.P. Time scales of Southern Ocean eddy equilibration. J. Phys. Oceanogr. 2016, 46, $2785-2805$. doi:10.1175/JPO-D-16-0041.1.

47. Pedlosky, J. The Equations for Geostrophic Motion in the Ocean. J. Phys. Oceanogr. 1984, 14, 448-456. doi:10.1175/15200485(1984)014<0448:TEFGMI>2.0.CO;2.

48. Grooms, I.; Julien, K.; Fox-Kemper, B. On the interactions between planetary geostrophy and mesoscale eddies. Dyn. Atmos. Ocean 2011, 51, 109-136. doi:10.1016/j.dynatmoce.2011.02.002.

49. Jamet, Q.; Deremble, B.; Wienders, N.; Uchida, T.; Dewar, W. On Wind-driven Energetics of Subtropical Gyres. J. Adv. Model. Earth Syst. 2021, 13, e2020MS002329. doi:10.1029/2020MS002329.

50. Marshall, J.; Adcroft, A.; Hill, C.; Perelman, L.; Heisey, C. A finite-volume, incompressible Navier Stokes model for studies of the ocean on parallel computers. J. Geophys. Res.: Oceans 1997, 102, 5753-5766. doi:10.1029/96JC02775.

51. Grooms, I.; Zanna, L. A note on 'Toward a stochastic parameterization of ocean mesoscale eddies'. Ocean Model. 2017, 113, 30-33. doi:10.1016/j.ocemod.2017.03.007.

52. Abernathey, R.; Squire, D.; Bourbeau, J.; Nicholas, T.; Bailey, S.; others. xhistogram: Fast, flexible, label-aware histograms for numpy and xarray, 2021.

53. Hirschi, J.J.M.; Frajka-Williams, E.; Blaker, A.T.; Sinha, B.; Coward, A.; Hyder, P.; Biastoch, A.; Böning, C.; Barnier, B.; Penduff, T.; others. Loop Current variability as trigger of coherent Gulf Stream transport anomalies. J. Phys. Oceanogr. 2019, 49, 2115-2132. doi:10.1175/JPO-D-18-0236.1.

54. Raymond, C.; Horton, R.M.; Zscheischler, J.; Martius, O.; AghaKouchak, A.; Balch, J.; Bowen, S.G.; Camargo, S.J.; Hess, J.; Kornhuber, K.; others. Understanding and managing connected extreme events. Nature climate change 2020, 10, 611-621. doi:10.1038/s41558-020-0790-4.

55. Gröger, M.; Dieterich, C.; Meier, H. Is interactive air sea coupling relevant for simulating the future climate of Europe? 2021. 56, 491-514. doi:10.1007/s00382-020-05489-8.

56. Randall, D.; Khairoutdinov, M.; Arakawa, A.; Grabowski, W. Breaking the cloud parameterization deadlock. Bulletin of the American Meteorological Society 2003, 84, 1547-1564. doi:10.1175/BAMS-84-11-1547.

57. Khairoutdinov, M.; Randall, D.; Demott, C.A. Simulations of the Atmospheric General Circulation Using a Cloud-Resolving Model as a Superparameterization of Physical Processes. J. Atmos. Sci. 2005, 62, 2136-2154. doi:10.1175/JAS3453.1.

58. Young, W.R. An Exact Thickness-Weighted Average Formulation of the Boussinesq Equations. J. Phys. Oceanogr. 2012, $42,692-707$. doi:10.1175/JPO-D-11-0102.1. 